\title{
ON VIRTUAL CABLING AND STRUCTURE OF 4-STRAND VIRTUAL PURE BRAID GROUP
}

\author{
VALERIY G. BARDAKOV AND JIE WU
}

\begin{abstract}
This article is dedicate to cabling on virtual braids. This construction gives a new generating set for the virtual pure braid group $V P_{n}$. Consequently we describe $V P_{4}$ as HNN-extension. As an application to classical braids, we find a new presentation of the Artin pure braid group $P_{4}$ in terms of the cabled generators.
\end{abstract}

\section{INTRODUCTION}

Cabling on classical braids has been used for establishing the fundamental connections between the homotopy groups and the theory of Brunnian braids [5] as well as a relationship between associators (for quasi-triangular quasi-Hopf algebras) and (a variant of) the Grothendieck-Teichmuller group [4]. In the paper [6], cabling for braids was used to study some properties of Burau representation. Similar operation (called naive cabling) on framed links has been explored in [8] with obtaining simplicial groups arising from link groups.

The purpose of this article is to explore cabling for virtual braids. Along the ideas in [7] on cabling for classical braids, one gets cabling operation for virtual pure braid group $V P_{n}$ that gives new generators for $V P_{n}$. More precisely, for $n \geq 3$, the group $V P_{n}$ is generated by the $n$-strand virtual braids obtained by taking $(k, l)$-cabling on the standard generators $\lambda_{1,2}$ and $\lambda_{2,1}$ of $V P_{2}$ together with adding trivial strands $n-k-l$ to the end for $1 \leq k \leq n-1$ and $2 \leq k+l \leq n$, where a $(k, l)$-cabling on a 2 -strand virtual braid means to take $k$-cabling on the first strand and $l$-cabling on the second strand.

Different from the classical situation [7] that the $n$-strand braids cabled from the standard generator $A_{1,2}$ for $P_{2}$ generates a free group of rank $n-1$, the subgroup of $V P_{n}$ generated by $n$-strand virtual braids cabled from $\lambda_{1,2}$ and $\lambda_{2,1}$, which is denoted by $T_{n-1}$, is no longer free for $n \geq 3$. For the first nontrivial case that $n=3$, a presentation of $T_{2}$ has been explored with producing a decomposition theorem for $V P_{3}$ using cabled generators [2].

Our main work in this article is to introduce a new generating set for $V P_{n}$, define a simplicial group $T_{*}$ and extend the results on $V P_{3}$ in [2] to $V P_{4}$. The main result is Theorem 5.12 that describe $V P_{4}$ as HNN-extension. As a consequence, we get a presentation for the group $T_{3}$ in Theorem 5.14. In the next article [3] we prove the

2010 Mathematics Subject Classification. 20F36, 55Q40, $18 \mathrm{G} 30$.

Key words and phrases. homotopy group, virtual braid group, simplicial group, virtual cabling. The main result is supported by the Russian Science Foundation grant N 16-11-10073. 
lifting theorem for the virtual braids. From this theorem follows that if we know the structure of $V P_{4}, T_{3}$ or $P_{4}$, then using degeneracy maps we can find the structure of $V P_{n}, T_{n}$ or $P_{n}$ for all bigger $n$.

The article is organized as follows. In Section 2.2, we give a review on braid groups and virtual braid groups. The simplicial structure on virtual pure braid groups will be discussed in Section 3. In Section 4, we discuss the cabling operation on classical pure braid group $P_{n}$ as subgroup $V P_{n}$. In particular, we give a new presentation of the Artin pure braid group $P_{4}$ in terms of the cabled generators in Proposition 4.1. We explore the structures of $V P_{3}$ and $V P_{4}$ in Section 5.

1.1. Acknowledgements. This article was written when the first author visited College of Mathematics and information Science Hebei Normal University. He thanks the administration for good working conditions. The authors would like to thank Roman Mikhailov for interesting ideas and useful discussion and Yu. Mikhal'chishina, who made the picture.

\section{BRAID AND VIRTUAL BRAID GROUPS}

2.1. Braid group. The braid group $B_{n}$ on $n$ strings is generated by $\sigma_{1}, \sigma_{2}, \ldots, \sigma_{n-1}$ and is defined by relations

$$
\begin{aligned}
& \sigma_{i} \sigma_{i+1} \sigma_{i}=\sigma_{i+1} \sigma_{i} \sigma_{i+1} \\
& \sigma_{i} \sigma_{j}=\sigma_{j} \sigma_{i},|i-j|>1 .
\end{aligned}
$$

Let $S_{n}, n \geq 1$ be the symmetric group which is generated by $\rho_{1}, \rho_{2}, \ldots, \rho_{n-1}$ and is defined by relations

$$
\begin{aligned}
& \rho_{i}^{2}=1, i=1,2, \ldots, n-1, \\
& \rho_{i} \rho_{i+1} \rho_{i}=\rho_{i+1} \rho_{i} \rho_{i+1}, i=1,2, \ldots, n-2, \\
& \rho_{i} \rho_{j}=\rho_{j} \rho_{i},|i-j|>1 .
\end{aligned}
$$

There is a homomorphism $B_{n} \rightarrow S_{n}$, which sends $\sigma_{i}$ to $\rho_{i}$. Its kernel is the pure braid group $P_{n}$. This group is generated by elements $A_{i, j}, 1 \leq i<j \leq n$, where

$$
\begin{gathered}
A_{i, i+1}=\sigma_{i}^{2}, \\
A_{i, j}=\sigma_{j-1} \sigma_{j-2} \ldots \sigma_{i+1} \sigma_{i}^{2} \sigma_{i+1}^{-1} \ldots \sigma_{j-2}^{-1} \sigma_{j-1}^{-1}, i+1<j \leq n,
\end{gathered}
$$

and is defined by relations (where $\varepsilon= \pm 1$ ):

$$
\begin{aligned}
& A_{i k}^{-\varepsilon} A_{k j} A_{i k}^{\varepsilon}=\left(A_{i j} A_{k j}\right)^{\varepsilon} A_{k j}\left(A_{i j} A_{k j}\right)^{-\varepsilon}, \\
& A_{k m}^{-\varepsilon} A_{k j} A_{k m}^{\varepsilon}=\left(A_{k j} A_{m j}\right)^{\varepsilon} A_{k j}\left(A_{k j} A_{m j}\right)^{-\varepsilon}, m<j, \\
& A_{i m}^{-\varepsilon} A_{k j} A_{i m}^{\varepsilon}=\left[A_{i j}^{-\varepsilon}, A_{m j}^{-\varepsilon}\right]^{\varepsilon} A_{k j}\left[A_{i j}^{-\varepsilon}, A_{m j}^{-\varepsilon}\right]^{-\varepsilon}, i<k<m, \\
& A_{i m}^{-\varepsilon} A_{k j} A_{i m}^{\varepsilon}=A_{k j}, \quad k<i, m<j \text { or } m<k,
\end{aligned}
$$

Here and further $[a, b]=a^{-1} b^{-1} a b$ is the commutator of $a$ and $b, a^{b}=b^{-1} a b$ is the conjugation of $a$ by $b$. 
There is an epimorphism of $P_{n}$ to $P_{n-1}$ what is removing of the $n$-th string. Its kernel $U_{n}=\left\langle A_{1 n}, A_{2 n}, \ldots, A_{n-1, n}\right\rangle$ is a free group of rank $n-1$ and $P_{n}=U_{n} \lambda P_{n-1}$ is a semi-direct product of $U_{n}$ and $P_{n-1}$. Hence,

$$
P_{n}=U_{n} \lambda\left(U_{n-1} \lambda\left(\ldots \lambda\left(U_{3} \lambda U_{2}\right)\right) \ldots\right),
$$

is a semi-direct product of free groups and $U_{2}=\left\langle A_{12}\right\rangle$ is the infinite cyclic group.

2.2. Virtual braid group. The virtual braid group $V B_{n}$ is generated by elements

$$
\sigma_{1}, \sigma_{2}, \ldots, \sigma_{n-1}, \rho_{1}, \rho_{2}, \ldots, \rho_{n-1},
$$

where $\sigma_{1}, \sigma_{2}, \ldots, \sigma_{n-1}$ generate the classical braid group $B_{n}$ and the elements $\rho_{1}$, $\rho_{2}, \ldots, \rho_{n-1}$ generate the symmetric group $S_{n}$. Hence, $V B_{n}$ is defined by relations of $B_{n}$, relations of $S_{n}$ and mixed relations:

$$
\begin{gathered}
\sigma_{i} \rho_{j}=\rho_{j} \sigma_{i},|i-j|>1, \\
\rho_{i} \rho_{i+1} \sigma_{i}=\sigma_{i+1} \rho_{i} \rho_{i+1} .
\end{gathered}
$$

As for the classical braid groups there exists the canonical epimorphism of $V P_{n}$ onto the symmetric group $V B_{n} \rightarrow S_{n}$ with the kernel called the virtual pure braid group $V P_{n}$. So we have a short exact sequence

$$
1 \rightarrow V P_{n} \rightarrow V B_{n} \rightarrow S_{n} \rightarrow 1 \text {. }
$$

Define the following elements in $V P_{n}$ :

$$
\begin{gathered}
\lambda_{i, i+1}=\rho_{i} \sigma_{i}^{-1}, \lambda_{i+1, i}=\rho_{i} \lambda_{i, i+1} \rho_{i}=\sigma_{i}^{-1} \rho_{i}, i=1,2, \ldots, n-1, \\
\lambda_{i j}=\rho_{j-1} \rho_{j-2} \ldots \rho_{i+1} \lambda_{i, i+1} \rho_{i+1} \ldots \rho_{j-2} \rho_{j-1}, \\
\lambda_{j i}=\rho_{j-1} \rho_{j-2} \ldots \rho_{i+1} \lambda_{i+1, i} \rho_{i+1} \ldots \rho_{j-2} \rho_{j-1}, 1 \leq i<j-1 \leq n-1 .
\end{gathered}
$$

It is shown in [1] that the group $V P_{n}(n \geq 2)$ admits a presentation with the generators $\lambda_{i j}, 1 \leq i \neq j \leq n$, and the following relations:

$$
\begin{aligned}
& \lambda_{i j} \lambda_{k l}=\lambda_{k l} \lambda_{i j}, \\
& \lambda_{k i} \lambda_{k j} \lambda_{i j}=\lambda_{i j} \lambda_{k j} \lambda_{k i},
\end{aligned}
$$

where distinct letters stand for distinct indices.

Like the classical pure braid groups, groups $V P_{n}$ admit a semi-direct product decompositions [1]: for $n \geq 2$, the $n$-th virtual pure braid group can be decomposed as

$$
V P_{n}=V_{n-1}^{*} \rtimes V P_{n-1}, n \geq 2,
$$

where $V_{n-1}^{*}$ is a subgroup of $V P_{n}, V_{1}^{*}=F_{2}, V P_{1}$ is supposed to be the trivial group. 


\section{Simplicial gROUPS $V P_{*}$ AND $T_{*}$}

3.1. Simplicial sets and simplicial groups. Recall the definition of simplicial groups (see [10, p. 300] or [5]). A sequence of sets $\mathcal{X}=\left\{X_{n}\right\}_{n \geq 0}$ is called a simplicial set if there are face maps:

$$
d_{i}: X_{n} \longrightarrow X_{n-1} \text { for } 0 \leq i \leq n
$$

and degeneracy maps

$$
s_{i}: X_{n} \longrightarrow X_{n+1} \text { for } 0 \leq i \leq n,
$$

that are satisfy the following simplicial identities:

(1). $d_{i} d_{j}=d_{j-1} d_{i}$ if $i<j$,

(2). $s_{i} s_{j}=s_{j+1} s_{i}$ if $i \leq j$,

(3). $d_{i} s_{j}=s_{j-1} d_{i}$ if $i<j$,

(4). $d_{j} s_{j}=i d=d_{j+1} s_{j}$,

(5). $d_{i} s_{j}=s_{j} d_{i-1}$ if $i>j+1$.

3.2. The cablings of virtual pure braid groups. By using the same ideas in the work [5, 7] on the classical braids, we have a simplcial group

$$
\mathrm{VAP}_{*}: \quad \ldots \underset{\stackrel{\rightleftarrows}{\rightleftarrows}}{\stackrel{\vec{\rightleftarrows}}{\rightleftarrows}} V P_{4} \underset{\rightleftarrows}{\rightleftarrows} V P_{3} \underset{\rightleftarrows}{\rightleftarrows} V P_{2} \rightleftarrows V P_{1}
$$

on pure virtual braid groups with $\mathrm{VAP}_{n}=V P_{n+1}$, the face homomorphism

$$
d_{i}: \mathrm{VAP}_{n}=V P_{n+1} \longrightarrow \mathrm{VAP}_{n-1}=V P_{n}
$$

given by deleting $(i+1)$ th strand for $0 \leq i \leq n$, and the degeneracy homomorphism

$$
s_{i}: \mathrm{VAP}_{n}=V P_{n+1} \longrightarrow \mathrm{VAP}_{n+1}=V P_{n+2}
$$

given by doubling the $(i+1)$ th strand for $0 \leq i \leq n$.

The idea of cabling is obtained from the geometric description, which can be regarded as the formal definition. See the Figure 1.

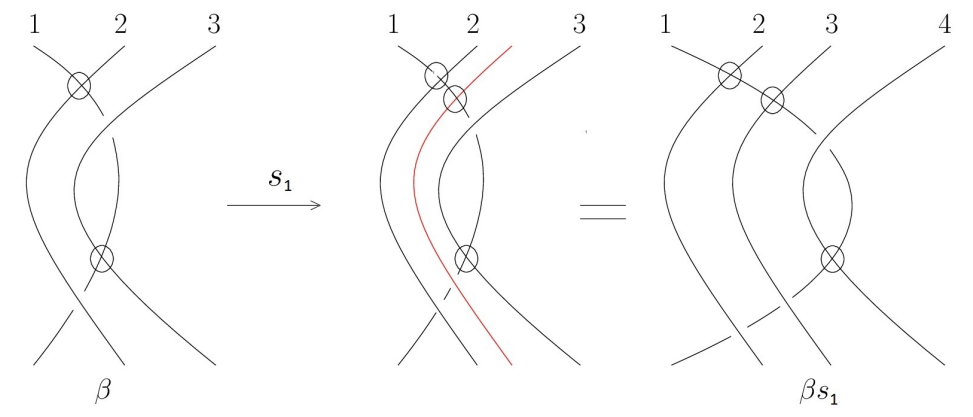

FiguRE 1. Degeneracy map $s_{1}$

The proof of the following proposition is straightforward. 
Proposition 3.1. The sequence of groups $\mathrm{VAP}_{*}$ with $\mathrm{VAP}_{n}=V P_{n+1}$ for $n \geq 0$ is a simplicial group under the faces $d_{i}$ : $\mathrm{VAP}_{n-1}=V P_{n} \longrightarrow \mathrm{VAP}_{n-2}=V P_{n-1}, 0 \leq i \leq$ $n-1$, and degeneracies $s_{i}: \mathrm{VAP}_{n-1}=V P_{n} \longrightarrow \mathrm{VAP}_{n}=V P_{n+1}, 0 \leq i \leq n-1$, given the group homomorphism with acting on the generators $\lambda_{k, l}$ and $\lambda_{l, k}, 1 \leq k<l \leq n$, of $V P_{n}$ by the rules

$$
\begin{gathered}
s_{i}\left(\lambda_{k, l}\right)= \begin{cases}\lambda_{k+1, l+1} & \text { for } i<k-1, \\
\lambda_{k, l+1} \lambda_{k+1, l+1} & \text { for } i=k-1, \\
\lambda_{k, l+1} & \text { for } \quad k-1<i<l-1, \\
\lambda_{k, l+1} \lambda_{k, l} & \text { for } i=l-1, \\
\lambda_{k, l} & \text { for } i>l-1,\end{cases} \\
s_{i}\left(\lambda_{l, k}\right)= \begin{cases}\lambda_{l+1, k+1} & \text { for } i<k-1, \\
\lambda_{l+1, k+1} \lambda_{l+1, k} & \text { for } i=k-1, \\
\lambda_{l+1, k} & \text { for } \quad k-1<i<l-1, \\
\lambda_{l, k} \lambda_{l+1, k} & \text { for } i=l-1, \\
\lambda_{l, k} & \text { for } i>l-1,\end{cases} \\
d_{i}\left(\lambda_{k, l}\right)= \begin{cases}\lambda_{k-1, l-1} & \text { for } \quad 0 \leq i<k-1, \\
1 & \text { for } i=k-1, \\
\lambda_{k, l-1} & \text { for } \quad k-1<i<l-1, \\
1 & \text { for } i=l, \\
\lambda_{k, l} & \text { for } \quad l-1<i \leq n-1,\end{cases} \\
d_{i}\left(\lambda_{l, k}\right)=\left\{\begin{array}{lll}
\lambda_{k-1, l-1} & \text { for } \quad 0 \leq i<k-1, \\
1 & \text { for } i=k-1, \\
\lambda_{l-1, k} & \text { for } \quad k-1<i<l-1, \\
1 & \text { for } i=l-1, \\
\lambda_{l, k} & \text { for } \quad l-1<i \leq n-1 .
\end{array}\right.
\end{gathered}
$$

Let $T_{*}$ be the smallest simplicial subgroup of $\mathrm{VAP}_{*}$ with the 1-simplex group $T_{1}=\mathrm{VAP}_{1}=V P_{2}$. It is routine to see that the group $T_{n}$ as a subgroup of $V P_{n+1}$ can be constructed recursively as follows:

$$
T_{0}=\{1\}, T_{1}=V P_{2}, \text { and } T_{n+1}=\left\langle s_{0}\left(T_{n}\right), s_{1}\left(T_{n}\right), \ldots, s_{n}\left(T_{n}\right)\right\rangle .
$$

Let

$$
\begin{aligned}
a_{k, n+1-k} & =s_{n-1} s_{n-2} \cdots s_{k} \hat{s}_{k-1} s_{k-2} \cdots s_{0} \lambda_{1,2}, \\
b_{k, n+1-k} & =s_{n-1} s_{n-2} \cdots s_{k} \hat{s}_{k-1} s_{k-2} \cdots s_{0} \lambda_{2,1}
\end{aligned}
$$


be the elements in $V P_{n+1}$ for $1 \leq k \leq n$. By direct computations, we have the following formulae.

$$
\begin{array}{r}
a_{n-k, k}=\left\{\begin{array}{lr}
\lambda_{1 n} \lambda_{2 n} \ldots \lambda_{n-1, n} & \text { for } k=1, \\
\lambda_{1 n} \lambda_{2 n} \ldots \lambda_{n-k, n} a_{n-k, k-1} & \text { for } 1<k<n, \\
\lambda_{1 n} a_{1, n-2} & \text { for } k=n-1,
\end{array}\right. \\
b_{n-k, k}=\left\{\begin{array}{lr}
\lambda_{n, n-1} \lambda_{n, n-2} \ldots \lambda_{n 1} & \text { for } k=1, \\
b_{n-k, k-1} \lambda_{n, n-k} \lambda_{n, n-k-1} \ldots \lambda_{n 1} & \text { for } 1<k<n, \\
b_{1, n-2} \lambda_{n 1} & \text { for } k=n-1,
\end{array}\right.
\end{array}
$$

Moreover the generators $\lambda_{i j}$ can be written in terms of $a_{k, l}$ and $b_{s, t}$ as follows:

$$
\begin{array}{r}
\lambda_{k n}=\left\{\begin{array}{lr}
a_{1, n-1} a_{1, n-2}^{-1} & \text { for } k=1, \\
a_{k-1, n-k} a_{k-1, n-k+1}^{-1} a_{k, n-k} a_{k, n-k-1}^{-1} & \text { for } 1<k<n, \\
a_{n-2,1} a_{n-2,2}^{-1} a_{n-1,1} & \text { for } k=n-1,
\end{array}\right. \\
\lambda_{n k}=\left\{\begin{array}{lr}
b_{1, n-2}^{-1} b_{1, n-1} & \text { for } k=1, \\
b_{k, n-k-1}^{-1} b_{k, n-k} b_{k-1, n-k+1}^{-1} b_{k-1, n-k} & \text { for } 1<k<n, \\
b_{n-1,1} b_{n-2,2}^{-1} b_{n-2,1} & \text { for } k=n-1,
\end{array}\right.
\end{array}
$$

From the above formulae, we have the following proposition.

Proposition 3.2. Consider $V P_{k}$ as a subgroup of $V P_{k+1}$ by adding a trivial strand in the end. Then

(1). The subgroup $T_{n-1}$ of $V P_{n}, n \geq 3$, is generated by elements $a_{k, l}, b_{k, l}, k+l=n$.

(2). The group $V P_{n}=\left\langle T_{1}, T_{2}, \ldots, T_{n-1}\right\rangle$ generated by $a_{k, l}$ and $b_{k, l}$ for $2 \leq k+l \leq$ $n, 1 \leq k, l \leq n-1$.

(3). $V P_{n+1}=\left\langle V P_{n}, s_{0} V P_{n}, s_{1} V P_{n}, \ldots, s_{n-1} V P_{n}\right\rangle$ for $n \geq 2$.

Let $c_{i j}=b_{i j} a_{i j}$. Put

$$
T_{i}^{c}=\left\langle c_{i j} \mid i+j=n-1\right\rangle, i=1,2, \ldots, n-1 .
$$

Notice that

$$
c_{1,1}=b_{1,1} a_{1,1}=\lambda_{2,1} \lambda_{1,2}=\sigma_{1}^{-1} \rho_{1} \rho_{1} \sigma_{1}^{-1}=\sigma_{1}^{-2}
$$

is a generator for $P_{2}$ as a subgroup of $V P_{2}$. The cabled braid $c_{i, j}$ lies in $P_{i+j+1} \leq$ $V P_{i+j+1}$. It is straightforward to see that the following proposition holds for classical braids.

Proposition 3.3. Consider $P_{k}$ as a subgroup of $P_{k+1}$ by adding a trivial strand in the end. Then

(1). The subgroup $T_{n-1}^{c}$ of $P_{n}, n \geq 3$, is generated by elements $c_{k, l}, k+l=n$.

(2). The group $P_{n}=\left\langle T_{1}^{c}, T_{2}^{c}, \ldots, T_{n-1}^{c}\right\rangle$ generated by $c_{k, l}$ for $2 \leq k+l \leq n, 1 \leq$ $k, l \leq n-1$.

(3). $P_{n+1}=\left\langle P_{n}, s_{0} P_{n}, s_{1} P_{n}, \ldots, s_{n-1} P_{n}\right\rangle$ for $n \geq 2$. 


\section{Cabling of the Classical PuRe BRAid Group}

In the present section we find a set of defining relations of $P_{4}$ in the generators $c_{i j}$ in Proposition 3.3 .

Proposition 4.1. The group $P_{4}$ is generated by elements

$$
c_{11}, c_{21}, c_{12}, c_{31}, c_{22}, c_{13}
$$

and is defined by relations (where $\varepsilon= \pm 1$ ):

$$
\begin{gathered}
c_{21}^{c_{11}^{\varepsilon}}=c_{21}, c_{12}^{c_{11}^{\varepsilon}}=c_{12}^{c_{21}^{-\varepsilon}}, c_{31}^{c_{11}^{\varepsilon}}=c_{31}, c_{22}^{c_{11}^{\varepsilon}}=c_{22}, c_{13}^{c_{11}^{\varepsilon}}=c_{13}^{c_{22}^{-\varepsilon}} \\
c_{31}^{c_{21}^{\varepsilon}}=c_{31}, c_{22}^{c_{21}^{\varepsilon}}=c_{22}^{c_{31}^{-\varepsilon}}, c_{13}^{c_{21}^{\varepsilon}}=c_{13}^{c_{22}^{\varepsilon} c_{31}^{-\varepsilon}} \\
c_{31}^{c_{12}^{\varepsilon}}=c_{31}, c_{13}^{c_{12}^{\varepsilon}}=c_{13}^{c_{31}^{-\varepsilon}} \\
c_{22}^{c_{12}^{-1}}=\left[c_{31}, c_{13}^{-1}\right]\left[c_{13}^{-1}, c_{22}\right] c_{22}\left[c_{21}^{2}, c_{12}^{-1}\right]=c_{13}^{c_{31}} c_{13}^{-c_{22}} c_{22}\left[c_{21}^{2}, c_{12}^{-1}\right] \\
c_{22}^{c_{12}}=\left[c_{12}, c_{21}^{-2}\right] c_{22}\left[c_{22}^{-3}, c_{13}\right]\left[c_{13}, c_{31}^{-1}\right]=\left[c_{12}, c_{21}^{-2}\right] c_{13}^{-c_{22}^{-2}} c_{22} c_{13}^{c_{31}} .
\end{gathered}
$$

Proof. Rewrite the generators $c_{i j}$ in the standard generators of $P_{4}$. We have

$$
\begin{gathered}
c_{11}=b_{11} a_{11}=\lambda_{21} \lambda_{12}=\sigma_{1}^{-1} \rho_{1} \rho_{1} \sigma_{1}^{-1}=\sigma_{1}^{-2}=A_{12}^{-1}, \\
c_{21}=b_{21} a_{21}=\lambda_{32}\left(\lambda_{31} \lambda_{13}\right) \lambda_{23}=\sigma_{2}^{-1} \lambda_{21} \lambda_{12} \sigma_{2}^{-1}=\sigma_{2}^{-1} A_{12}^{-1} \sigma_{2}^{-1}=\sigma_{2}^{-2} \sigma_{2} A_{12}^{-1} \sigma_{2}^{-1}=A_{23}^{-1} A_{13}^{-1}, \\
c_{12}=b_{12} a_{12}=\lambda_{21}\left(\lambda_{31} \lambda_{13}\right) \lambda_{12}=\sigma_{1}^{-1} \rho_{1} \rho_{2} \lambda_{21} \lambda_{12} \rho_{2} \rho_{1} \sigma_{1}^{-1}=\sigma_{1}^{-1} \rho_{1} \lambda_{31} \lambda_{13} \rho_{1} \sigma_{1}^{-1}= \\
=\sigma_{1}^{-1} \lambda_{32} \lambda_{23} \sigma_{1}^{-1}=\sigma_{1}^{-1} \sigma_{2}^{-1} \sigma_{2}^{-1} \sigma_{1}^{-1}=\sigma_{1}^{-1} A_{23}^{-1} \sigma_{1}^{-1}=\left(\sigma_{1}^{-1} A_{23}^{-1} \sigma_{1}\right) A_{12}^{-1}=A_{13}^{-1} A_{12}^{-1} .
\end{gathered}
$$

And analogously,

$$
c_{31}=A_{34}^{-1} A_{24}^{-1} A_{14}^{-1}, c_{22}=A_{24}^{-1} A_{14}^{-1} A_{23}^{-1} A_{13}^{-1}, c_{13}=A_{14}^{-1} A_{13}^{-1} A_{12}^{-1} .
$$

In particular, we see that

$$
\begin{gathered}
P_{2}=T_{1}^{c}=\left\langle A_{12}\right\rangle \\
P_{3}=\left\langle T_{1}^{c}, T_{2}^{c}\right\rangle=\left\langle c_{11}, c_{21}, c_{12}\right\rangle \\
P_{4}=\left\langle T_{1}^{c}, T_{2}^{c}, T_{3}^{c}\right\rangle=\left\langle c_{11}, c_{21}, c_{12}, c_{31}, c_{22}, c_{13}\right\rangle .
\end{gathered}
$$

To find a set of defining relations, express the old generators in the new one:

$$
\begin{gathered}
A_{12}=c_{11}^{-1}, A_{13}=c_{11} c_{12}^{-1}, A_{23}=c_{12} c_{21}^{-1} c_{11}^{-1} . \\
A_{14}=c_{12} c_{13}^{-1}, A_{24}=c_{13} c_{12}^{-1} c_{21} c_{22}^{-1}, A_{34}=c_{22} c_{21}^{-1} c_{31}^{-1} .
\end{gathered}
$$

Rewriting the set of defining relations of $P_{4}$ in the new generators we will find the set of defining relations.

Let us prove the formula for $c_{22}^{c_{12}^{-1}}$ and for $c_{22}^{c_{12}}$, assuming that all other formulas are true. Proofs for all others not difficult. Take the relation

$$
A_{13}^{-1} A_{24} A_{13}=\left[A_{14}^{-1}, A_{34}^{-1}\right] A_{24}\left[A_{34}^{-1}, A_{14}^{-1}\right] .
$$

In the new generators this relation after cancellations has the form

$$
\left(c_{13} c_{12}^{-1} c_{21} c_{22}^{-1}\right)^{c_{11}}=c_{13}^{-1} c_{22} c_{21}^{-1} c_{31}^{-1} c_{13} c_{12}^{-1} c_{31} c_{21} c_{22}^{-1} c_{13} c_{31}^{-1} c_{13}^{-1} c_{31} c_{21} c_{22}^{-1} c_{13} \text {. }
$$


Using the formulas of conjugating by $c_{11}^{-1}$ we get

$$
c_{22} c_{13} c_{22}^{-1} c_{21} c_{12}^{-1} c_{22}^{-1}=c_{13}^{-1} c_{22}\left(c_{21}^{-1} c_{31}^{-1} c_{13}\right) c_{12}^{-1} c_{31} c_{21} c_{22}^{-1} c_{13} c_{31}^{-1} c_{13}^{-1} c_{31}\left(c_{21} c_{22}^{-1} c_{13}\right) .
$$

Rewrite the term in the brackets in the form

$$
\begin{aligned}
& c_{21}^{-1} c_{31}^{-1} c_{13}=\left(c_{31}^{-1} c_{13}\right)^{c_{21}} c_{21}^{-1}=c_{22}^{-1} c_{13} c_{22} c_{31}^{-1} c_{21}^{-1}, \\
& c_{21} c_{22}^{-1} c_{13}=\left(c_{22}^{-1} c_{13}\right)^{c_{21}^{-1}} c_{21}=c_{31}^{-1} c_{13} c_{22}^{-1} c_{31} c_{21},
\end{aligned}
$$

we get

$$
\left(c_{13} c_{22}^{-1} c_{21}\right) c_{12}^{-1} c_{22}^{-1}=\left(c_{31}^{-1} c_{21}^{-1} c_{12}^{-1} c_{31}\right) c_{21}\left(c_{22}^{-1} c_{13} c_{31}^{-1} c_{22}^{-1} c_{31} c_{21}\right) .
$$

Using the conjugation rules, rewrite the term in the brackets in the form

$$
\begin{gathered}
c_{13} c_{22}^{-1} c_{21}=c_{21}\left(c_{13} c_{22}^{-1}\right)^{c_{21}}=c_{21} c_{31} c_{22}^{-1} c_{13} c_{31}^{-1}, c_{31}^{-1} c_{21}^{-1} c_{12}^{-1} c_{31}=\left(c_{21}^{-1} c_{12}^{-1}\right)^{c_{31}}=c_{21}^{-1} c_{12}^{-1}, \\
c_{22}^{-1} c_{13} c_{31}^{-1} c_{22}^{-1} c_{31} c_{21}=c_{21}\left(c_{22}^{-1} c_{13} c_{31}^{-1} c_{22}^{-1} c_{31}\right)^{c_{21}}=c_{21} c_{31} c_{22}^{-2} c_{13} c_{22} c_{31}^{-1} c_{22}^{-1},
\end{gathered}
$$

then

$$
c_{21} c_{31} c_{22}^{-1} c_{13} c_{31}^{-1} c_{12}^{-1}=c_{21}^{-1} c_{12}^{-1} c_{21}^{2} c_{31} c_{22}^{-2} c_{13} c_{22} c_{31}^{-1} \text {. }
$$

Conjugating both sides by $c_{31}$ and using the fact that it commutes with $c_{12}$ and $c_{21}$, we get

$$
c_{21}\left(c_{22}^{-1} c_{13} c_{12}^{-1}\right)=c_{21}^{-1} c_{12}^{-1} c_{21}^{2} c_{22}^{-2} c_{13} c_{22} .
$$

Transforming the expression in the brackets, we get

$$
c_{21} c_{12}^{-1} c_{22}^{-c_{12}^{-1}} c_{31}^{-1} c_{13} c_{31}=c_{21}^{-1} c_{12}^{-1} c_{21}^{2} c_{22}^{-2} c_{13} c_{22} \text {. }
$$

Multiply both sides to $c_{21}^{-2} c_{12} c_{21}$ on the left ant to $c_{31}^{-1} c_{13}^{-1} c_{31}$ on the right we get

$$
\left[c_{21}^{2}, c_{12}^{-1}\right] c_{22}^{-c_{12}^{-1}}=c_{22}^{-1}\left[c_{22}, c_{13}^{-1}\right]\left[c_{13}^{-1}, c_{31}\right]
$$

From this relation follows that

$$
c_{22}^{c_{12}^{-1}}=\left[c_{31}, c_{13}^{-1}\right]\left[c_{13}^{-1}, c_{22}\right] c_{22}\left[c_{21}^{2}, c_{12}^{-1}\right]
$$

To find conjugation formula $c_{22}^{c_{12}}$, we are using (4.1)

$$
c_{21} c_{22}^{-1} c_{13} c_{12}^{-1}=c_{21}^{-1} c_{12}^{-1} c_{21}^{2}\left(c_{22}^{-2} c_{13} c_{22}\right) \text {. }
$$

Since

then

$$
\left(c_{22}^{-1} c_{13}\right)^{c_{11}^{-1}}=c_{22}^{-2} c_{13} c_{22},
$$

$$
c_{21}\left(c_{22}^{-1} c_{13}\right) c_{12}^{-1}=c_{21}^{-1} c_{12}^{-1} c_{21}^{2}\left(c_{22}^{-1} c_{13}\right)^{c_{11}^{-1}} \text {. }
$$

Multiply both sides to $c_{21}^{-2} c_{12} c_{21}$ to the left:

$$
\left[c_{21}^{2}, c_{12}^{-1}\right]\left(c_{22}^{-1} c_{13}\right)^{c_{12}^{-1}}=\left(c_{22}^{-1} c_{13}\right)^{c_{11}^{-1}} \Leftrightarrow\left(c_{22}^{-1} c_{13}\right)^{c_{12}^{-1}}=\left[c_{12}^{-1}, c_{21}^{2}\right]\left(c_{22}^{-1} c_{13}\right)^{c_{11}^{-1}} .
$$

Using the relation

$$
\left[c_{12}^{-1}, c_{21}^{2}\right]=\left[c_{12}^{-1}, c_{11}^{-2}\right]
$$

we have

$$
c_{22}^{-1} c_{13} c_{12}^{-1}=c_{11}^{2} c_{12}^{-1} c_{11}^{-1}\left(c_{22}^{-1} c_{13}\right) c_{11}^{-1}
$$


Multiply both sides to $c_{12}$ on the right

$$
c_{22}^{-1} c_{13}=c_{11}^{2} c_{12}^{-1}\left(c_{11}^{-1} c_{22}^{-1} c_{13}\right) c_{11}^{-1} c_{12} .
$$

Rewrite expression in the brackets

$$
c_{11}^{-1} c_{22}^{-1} c_{13}=\left(c_{22}^{-1} c_{13}\right)^{c_{11}} c_{11}^{-1}=\left(c_{13} c_{22}^{-1}\right) c_{11}^{-1},
$$

then

$$
c_{22}^{-1} c_{13}=c_{11}^{2} c_{12}^{-1}\left(c_{13} c_{22}^{-1}\right) c_{11}^{-2} c_{12} \Leftrightarrow c_{22}^{-1} c_{13}=c_{11}^{2} c_{13}^{c_{12}} c_{22}^{-c_{12}} c_{12}^{-1} c_{11}^{-2} c_{12} .
$$

Multiply both sides to $c_{13}^{-c_{12}} c_{11}^{-2}$ on the left and to $c_{12}^{-1} c_{11}^{2} c_{12}$ on the right, we get

$$
c_{13}^{-c_{12}}\left(c_{11}^{-2} c_{22}^{-1} c_{13}\right) c_{12}^{-1} c_{11}^{2} c_{12}=c_{22}^{-c_{12}} .
$$

Find the expression in the brackets

$$
c_{11}^{-2} c_{22}^{-1} c_{13}=\left(c_{22}^{-1} c_{13}\right)^{c_{11}^{2}} c_{11}^{-2}=c_{22}^{-1} c_{13}^{c_{22}^{-2}} c_{11}^{-2} .
$$

Then

Using the relations

$$
c_{13}^{-c_{12}} c_{22}^{-1} c_{13}^{c_{22}^{-2}}\left[c_{11}^{2}, c_{12}\right]=c_{22}^{-c_{12}}
$$

$$
c_{13}^{-c_{12}}=c_{13}^{-c_{31}^{-1}},\left[c_{11}^{2}, c_{12}\right]=\left[c_{21}^{-2}, c_{12}\right],
$$

we get

$$
c_{13}^{-c_{31}^{-1}} c_{22}^{-1} c_{13}^{c_{22}^{-2}}\left[c_{21}^{-2}, c_{12}\right]=c_{22}^{-c_{12}} .
$$

from this relation follows the need relation.

4.1. Decomposition of $P_{4}$. In the paper [7] was proved that the Milnor simplicial group $F\left[S^{1}\right]$ is embedded into the simplicial group $A P_{*}$. The main problem in this theorem is the proof that groups $T_{n}^{c}, n=2,3, \ldots$, are free. To do it the authors used some Lie algebras. In this section we prove, that $T_{2}^{c}$ and $T_{3}^{c}$ are free groups using group-theoretical methods. Note, that $T_{1}^{c}$ is infinite cyclic.

From Proposition 4.1 follows that $P_{3}$ has the following presentation

$$
P_{3}=\left\langle c_{11}, c_{21}, c_{12} \| c_{21}^{c_{11}^{\varepsilon}}=c_{21}, c_{12}^{c_{11}^{\varepsilon}}=c_{12}^{c_{21}^{-\varepsilon}}\right\rangle .
$$

Hence, $P V_{3}=T_{2}^{c} \lambda \mathbb{Z}$, where $T_{2}=\left\langle c_{21}, c_{12}\right\rangle$ is a free group and $\mathbb{Z}=\left\langle c_{11}\right\rangle$.

To prove that $T_{3}^{c}$ is free, define a homomorphism of $P_{4}$ onto free abelian group of rank 2:

$$
\varphi: P_{4} \rightarrow\langle x, y \| x y=y x\rangle=\mathbb{Z}^{2}
$$

by the rule:

$$
\varphi\left(c_{11}\right)=x, \varphi\left(c_{21}\right)=y, \varphi\left(c_{12}\right)=e, \varphi\left(c_{31}\right)=e, \varphi\left(c_{22}\right)=e, \varphi\left(c_{13}\right)=e,
$$

where $e$ is the unit element of abelaian group.

Note that subgroup of $P_{4}$ that is generated by $c_{11}$ and $c_{21}$ is free abelian of rank 2 . Hence, for the short exact sequens

$$
1 \rightarrow \operatorname{Ker}(\varphi) \rightarrow P_{4} \rightarrow \mathbb{Z}^{2} \rightarrow 1
$$


there exist a section $s: \mathbb{Z}^{2} \rightarrow P_{4}, s(x)=a_{11}, s(y)=a_{21}$ and we have decomposition $P_{4}=\operatorname{Ker}(\varphi) \lambda \mathbb{Z}^{2}$ of $P_{4}$ into a semi-direct product.

Let us find a set of generators and defining relations for $\operatorname{Ker}(\varphi)$. Put

$$
\Lambda=\left\{c_{11}^{k} c_{21}^{l} \mid k, l \in \mathbb{Z}\right\}
$$

is a set of coset representatives of $P_{4}$ by $s\left(\mathbb{Z}^{2}\right)$. Then $\operatorname{Ker}(\varphi)$ is generated by elements

$$
c_{12}^{\lambda}, c_{31}^{\lambda}, c_{22}^{\lambda}, c_{13}^{\lambda} \text {, where } \lambda \in \Lambda \text {. }
$$

Using the following defining relations of $P_{4}$ :

$$
\begin{gathered}
c_{21}^{c_{11}^{\varepsilon}}=c_{21}, c_{12}^{c_{11}^{\varepsilon}}=c_{12}^{c_{21}^{-\varepsilon}}, c_{31}^{c_{11}^{\varepsilon}}=c_{31}, c_{22}^{c_{11}^{\varepsilon}}=c_{22}, c_{13}^{c_{11}^{\varepsilon}}=c_{13}^{c_{22}^{-\varepsilon}}, \\
c_{31}^{c_{21}^{\varepsilon}}=c_{31}, c_{22}^{c_{21}^{\varepsilon}}=c_{22}^{c_{31}^{-\varepsilon}}, c_{13}^{c_{21}^{\varepsilon}}=c_{13}^{c_{22}^{\varepsilon} c_{31}^{-\varepsilon}},
\end{gathered}
$$

rewrite the generators of $\operatorname{Ker}(\varphi)$ in the form

$$
c_{12}^{c_{11}^{k} c_{21}^{l}}=c_{12}^{c_{21}^{l-k}}, c_{31}^{c_{11}^{k} c_{21}^{l}}=c_{31}, c_{22}^{c_{11}^{k} c_{21}^{l}}=c_{22}^{c_{31}^{-l}}, c_{13}^{c_{11}^{k} c_{21}^{l}}=c_{13}^{c_{22}^{l-k} c_{31}^{-l}}
$$

Hence, $\operatorname{Ker}(\varphi)$ is generated by $c_{31}, c_{22}, c_{13}$ and infinite set $c_{12}^{c_{21}^{m}}, m \in \mathbb{Z}$. For simplicity we will denote $d_{m}=c_{12}^{c_{21}^{m}}$.

To find a set of defining relations of $\operatorname{Ker}(\varphi)$, we take the last relations of $P_{4}$ :

$$
\begin{gathered}
c_{31}^{c_{12}}=c_{31}, c_{13}^{c_{12}}=c_{13}^{c_{31}^{-1}} . \\
c_{22}^{c_{12}^{-1}}=c_{13}^{c_{31}} c_{13}^{-c_{22}} c_{22}\left[c_{21}^{2}, c_{12}^{-1}\right] .
\end{gathered}
$$

For simplicity, instead the last relation take relation (4.1):

$$
c_{21} c_{22}^{-1} c_{13} c_{12}^{-1}=c_{21}^{-1} c_{12}^{-1} c_{21}^{2}\left(c_{22}^{-2} c_{13} c_{22}\right),
$$

which is equivalent to the last one. Conjugating these relations by coset representatives $\lambda \in \Lambda$, we get a set of defining relations for $\operatorname{Ker}(\varphi)$.

1) Conjugating the relation $c_{12}^{-1} c_{31} c_{12}=c_{31}$ by $c_{11}^{k} c_{21}^{l}$, we get

$$
c_{12}^{-c_{21}^{l-k}} c_{31} c_{12}^{c_{21}^{l-k}}=c_{31} \text {. }
$$

Put $m=l-k$, we get the set of relations

$$
d_{m}^{-1} c_{31} d_{m}=c_{31}, m \in \mathbb{Z} .
$$

2) Conjugating the relation $c_{12}^{-1} c_{13} c_{12}=c_{31} c_{13} c_{31}^{-1}$ by $c_{11}^{k} c_{21}^{l}$, we get

$$
c_{12}^{-c_{21}^{l-k}} c_{13}^{c_{22}^{l-k}} c_{31}^{-l} c_{12}^{c_{21}^{l-k}}=c_{31} c_{13}^{c_{22}^{l-k}} c_{31}^{-l} c_{31}^{-1} \text {. }
$$

Conjugating this relation by $c_{31}^{l}$ and put $m=l-k$ we get the set of relations

$$
d_{m}^{-1} c_{13}^{c_{22}^{m}} d_{m}=c_{13}^{c_{22}^{m} c_{31}^{-1}}, m \in \mathbb{Z}
$$

3) Conjugating the relation $c_{22}^{-1} c_{13} c_{12}^{-1}=c_{12}^{-c_{21}^{2}} c_{22}^{-2} c_{13} c_{22}$ by $c_{11}^{k} c_{21}^{l}$, we get

$$
c_{22}^{-c_{31}^{-l}} c_{13}^{c_{22}^{l-k} c_{31}^{-l}} c_{12}^{-c_{21}^{l-k}}=c_{12}^{-c_{21}^{l-k+2}}\left(c_{22}^{-c_{31}^{-l}}\right)^{-2} c_{13}^{c_{22}^{l-k}} c_{31}^{-l} c_{22}^{c_{31}^{-l}} \text {. }
$$


Conjugating this relation by $c_{31}^{l}$ and put $m=l-k$ we get the set of relations

$$
c_{22}^{-1} c_{13}^{c_{22}^{l-k}} d_{m}^{-1}=d_{m+2}^{-1} c_{22}^{2} c_{13}^{c_{22}^{m}} c_{22}
$$

Hence, we prove

Lemma 4.2. $\operatorname{Ker}(\varphi)$ is generated by

$$
c_{31}, c_{22}, c_{13}, d_{m}, m \in \mathbb{Z}
$$

and is defined by relations

1) $d_{m}^{-1} c_{31} d_{m}=c_{31}$,

2) $d_{m}^{-1} c_{13}^{c_{22}^{m}} d_{m}=c_{13}^{c_{22}^{m} c_{31}^{-1}}$

3) $d_{m+2}=c_{22}^{-1} c_{13}^{c_{22}^{m+1}} d_{m} c_{13}^{-c_{22}^{m}} c_{22}$

for $m \in \mathbb{Z}$.

From the set of relations 3) we express all generators $d_{m}, m \neq 0,1$ as words in the generators

$$
d_{0}, d_{1}, c_{31}, c_{22}, c_{13}
$$

If $m=2 m_{1} \geq 0$, then from 3) we have

$$
d_{m}=c_{22}^{-m_{1}} c_{13}^{c_{22}^{m_{1}}} c_{13}^{c_{22}^{m_{1}-1}} \ldots c_{13}^{c_{22}} d_{0} c_{13}^{-1} c_{13}^{-c_{22}} \ldots c_{13}^{-c_{22}^{m_{1}-1}} c_{22}^{m_{1}} .
$$

If $m=2 m_{1}<0$, then rewrite 3 ) in the form

$$
d_{m}=c_{22} c_{13}^{-c_{22}^{m+2}} d_{m+2} c_{13}^{c_{22}^{m+1}} c_{22}^{-1}
$$

and by induction we get

$$
d_{m}=c_{22}^{-m_{1}} c_{13}^{-c_{22}^{-\left(m_{1}+1\right)}} c_{13}^{c_{22}^{-\left(m_{1}+2\right)}} \ldots c_{13}^{-c_{22}^{-1}} c_{13}^{-1} d_{0} c_{13}^{c_{22}^{-1}} c_{13}^{c_{22}^{-2}} \ldots c_{13}^{c_{22}^{-m_{1}}} c_{22}^{m_{1}} .
$$

Put these formulas into relations 2), we get

Lemma 4.3. The set of relations 2) for the even indexes $m=2 m_{1}$ is equivalent to the following set of relations:

$$
\left(c_{13}^{\left(c_{13} c_{22}\right)^{m_{1}}}\right)^{d_{0}}=c_{13}^{c_{22}^{m} c_{31}^{-1} c_{22}^{-m}\left(c_{22} c_{13}\right)^{m_{1}}} .
$$

Now consider the odd indexes. If $m=2 m_{1}+1>0$, then from 3) we have

$$
d_{m}=c_{22}^{-m_{1}} c_{13}^{c_{22}^{m_{1}+1}} c_{13}^{c_{22}^{m_{1}}} \ldots c_{13}^{c_{22}^{2}} d_{1} c_{13}^{-c_{22}} c_{13}^{-c_{22}^{2}} \ldots c_{13}^{-c_{22}^{m_{1}}} c_{22}^{m_{1}} \text {. }
$$

If $m=2 m_{1}+1<0$, then

$$
d_{m}=c_{22}^{-m_{1}} c_{13}^{-c_{22}^{m_{1}+2}} c_{13}^{-c_{22}^{m_{1}}+3} \ldots c_{13}^{-c_{22}^{-1}} c_{13}^{-1} c_{13}^{-c_{22}} d_{1} c_{13} c_{13}^{c_{22}^{-1}} \ldots c_{13}^{-c_{22}^{m_{1}+1}} c_{22}^{m_{1}} .
$$

Put these formulas into relations 2), we get 
Lemma 4.4. The set of relations 2) for the odd indexes $m=2 m_{1}+1$ is equivalent to the following set of relations:

$$
\left(c_{13}^{\left(c_{22} c_{13}\right)^{m_{1}-1} c_{22}^{2}}\right)^{d_{1}}=c_{13}^{c_{22}^{m} c_{31}^{-1} c_{22}^{-(m-1)}\left(c_{13} c_{22}\right)^{m_{1}}} .
$$

Considering the relations 1 ) and input the expressions for $d_{m}$ into these relations we get.

Lemma 4.5. The set of relations 1) is equivalent to the union of the following sets of relations:

if $m=2 m_{1}$ is even, then

$$
\left(c_{31}^{c_{22}^{-m}\left(c_{13} c_{22}\right)^{m_{1}}}\right)^{d_{0}}=c_{31}^{c_{22}^{-m}\left(c_{22} c_{13}\right)^{m_{1}}}
$$

if $m=2 m_{1}+1$ is odd, then

$$
\left(c_{31}^{c_{22}^{-(m+1)}\left(c_{22} c_{13}\right)^{m_{1}} c_{22}^{2}}\right)^{d_{1}}=c_{31}^{c_{22}^{-(m-1)}\left(c_{13} c_{22}\right)^{m_{1}}}
$$

Hence we have proven

Proposition 4.6. $\operatorname{Ker}(\varphi)$ is generated by

$$
c_{31}, c_{22}, c_{13}, d_{0}, d_{1}
$$

and is defined by relations from Lemmas 4.3 - 4.5 .

Now we are going to prove that $\operatorname{Ker}(\varphi)$ is two consequent HNN-extensions of the group $T_{3}^{c}=\left\langle c_{31}, c_{22}, c_{13}\right\rangle$. For this define subgroups $A_{0}, B_{0}, A_{1}, B_{1}$ of $G$. Let $m=2 m_{1}$ is even number, then $A_{0}$ is generated by elements

$$
c_{13}^{\left(c_{13} c_{22}\right)^{m_{1}}}, c_{31}^{c_{22}^{-m}\left(c_{13} c_{22}\right)^{m_{1}}}
$$

$B_{0}$ is generated by elements

$$
c_{13}^{c_{22}^{m} c_{31}^{-1} c_{22}^{-m}\left(c_{22} c_{13}\right)^{m_{1}}}, c_{31}^{c_{22}^{-m}\left(c_{22} c_{13}\right)^{m_{1}}} .
$$

Define a map $\psi_{0}: A_{0} \rightarrow B_{0}$ on the generators:

$$
c_{13}^{\left(c_{13} c_{22}\right)^{m_{1}}} \rightarrow c_{13}^{c_{22}^{m} c_{31}^{-1} c_{22}^{-m}\left(c_{22} c_{13}\right)^{m_{1}}}, c_{31}^{c_{22}^{-m}\left(c_{13} c_{22}\right)^{m_{1}}} \rightarrow c_{31}^{c_{22}^{-m}\left(c_{22} c_{13}\right)^{m_{1}}} .
$$

From Lemmas 4.3, 4.5 follows that $\psi_{0}$ is induced conjugation by $d_{0}$ in $\operatorname{Ker}(\varphi)$ and hence is an isomorphism.

Analogously, let $m=2 m_{1}+1$ is odd number, then $A_{1}$ is generated by elements

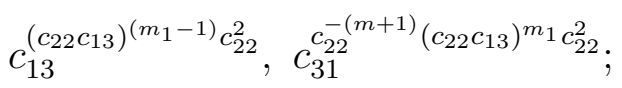

$B_{1}$ is generated by elements

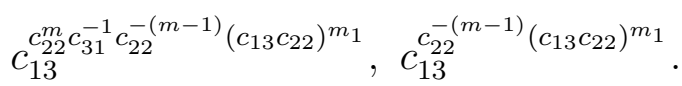


Define a map $\psi_{1}: A_{1} \rightarrow B_{1}$ on the generators:

$$
c_{13}^{\left(c_{22} c_{13}\right)^{\left(m_{1}-1\right)} c_{22}^{2}} \rightarrow c_{13}^{c_{22}^{m} c_{31}^{-1} c_{22}^{-(m-1)}\left(c_{13} c_{22}\right)^{m_{1}}}, c_{31}^{c_{22}^{-(m+1)}\left(c_{22} c_{13}\right)^{m_{1}} c_{22}^{2}} \rightarrow c_{13}^{c_{22}^{-(m-1)}\left(c_{13} c_{22}\right)^{m_{1}}} .
$$

From Lemmas 4.4, 4.5 follows that $\psi_{1}$ is induced conjugation by $d_{1}$ in $\operatorname{Ker}(\varphi)$ and hence is an isomorphism. In these notations we have

Theorem 4.7. $\operatorname{Ker}(\varphi)$ is two consequent $H N N$-extensions with the base group $T_{3}^{c}$ :

$$
\operatorname{Ker}(\varphi)=\left\langle T_{3}^{c}, d_{0}, d_{1} \| d_{0}^{-1} A_{0} d_{0}=B_{0}, \psi_{0} ; d_{1}^{-1} A_{1} d_{1}=B_{1}, \psi_{1}\right\rangle .
$$

Corollary 4.8. The group $T_{3}^{c}=\left\langle c_{31}, c_{22}, c_{13}\right\rangle$ is free of rank 3 .

Proof. The group $T_{3}^{c}$ is a subgroup of $\operatorname{Ker}(\varphi)$. From Theorem 4.7 follows that all relations of $\operatorname{Ker}(\varphi)$ are define $\operatorname{Ker}(\varphi)$ as HNN-extensions, hence $T_{3}^{c}$ does not have defining relations.

\section{Structure of $V P_{3}$ AND $V P_{4}$}

The main purpose of this section find sets of defining relations for $T_{2}$ and $T_{3}$. Note that $V P_{3}$ contains $T_{2}$ and has no commutativity relations, $V P_{4}$ contains $T_{3}$ and has commutativity relations, Relations of $T_{n}$ for $n>3$ one can find using degeneracy maps $s_{i}$.

5.1. The group $V P_{3}$. In the generators

$$
\lambda_{12}, \lambda_{21}, \lambda_{13}, \lambda_{23}, \lambda_{31}, \lambda_{23}
$$

$V P_{3}$ is defined by the following 6 relations

$$
\begin{aligned}
& \lambda_{12} \lambda_{13} \lambda_{23}=\lambda_{23} \lambda_{13} \lambda_{12}, \quad \lambda_{21} \lambda_{23} \lambda_{13}=\lambda_{13} \lambda_{23} \lambda_{21}, \lambda_{13} \lambda_{12} \lambda_{32}=\lambda_{32} \lambda_{12} \lambda_{13}, \\
& \lambda_{31} \lambda_{32} \lambda_{12}=\lambda_{12} \lambda_{32} \lambda_{31}, \quad \lambda_{23} \lambda_{21} \lambda_{31}=\lambda_{31} \lambda_{21} \lambda_{23}, \quad \lambda_{32} \lambda_{31} \lambda_{21}=\lambda_{21} \lambda_{31} \lambda_{32} .
\end{aligned}
$$

In the generators

$$
a_{11}, b_{11}, a_{21}, a_{12}, b_{21}, b_{12}
$$

$V P_{3}$ is defined by the following 6 relations

$$
\begin{aligned}
& {\left[a_{21}, a_{12}\right]=1, b_{11} a_{11} a_{21} a_{11}^{-1}=a_{21} b_{11}, a_{12} b_{21} b_{12}^{-1} b_{11}=b_{21} b_{12}^{-1} b_{11} a_{11} a_{12} a_{11}^{-1},} \\
& b_{11}^{-1} b_{21} b_{11} a_{11}=a_{11} b_{21}, a_{11} a_{12}^{-1} a_{21} b_{12}=b_{11}^{-1} b_{12} b_{11} a_{11} a_{12}^{-1} a_{21}, \quad\left[b_{21}, b_{12}\right]=1 .
\end{aligned}
$$

In the paper [2] was found the following decomposition of $V P_{3}$.

Proposition 5.1. ([2]) The group $V P_{3}$ is generated by elements

$$
a_{11}, c_{11}, a_{21}, a_{12}, b_{21}, b_{12}
$$

and is defined by relations

$$
\begin{gathered}
{\left[a_{21}, a_{12}\right]=\left[b_{21}, b_{12}\right]=1,} \\
a_{21}^{c_{11}}=a_{21}, b_{21}^{c_{11}}=b_{21}, b_{12}^{c_{11}}=b_{12}^{a_{21}^{-1} a_{12}}, a_{12}^{c_{11}}=a_{12}^{b_{12} a_{21}^{-1} a_{12} b_{21}^{-1},},
\end{gathered}
$$

i. e. $V P_{3}=\left\langle T_{2}, c_{11}\right\rangle *\left\langle a_{11}\right\rangle,\left\langle T_{2}, c_{11}\right\rangle=T_{2} \lambda\left\langle c_{11}\right\rangle$. 
In this proposition $c_{11}$ acts on $b_{12}$ and $a_{12}$ by different manner. Let us show that in fact these actions are equel. Indeed, since $a_{21}^{-1} a_{12}=a_{12} a_{21}^{-1}$, then

$$
a_{12}^{c_{11}}=a_{12}^{b_{12} a_{12} a_{21}^{-1} b_{21}^{-1}} \Leftrightarrow a_{12}^{c_{11}}=a_{12}^{c_{12} c_{21}^{-1}} .
$$

Similarly, rewrite the conjugation rule

$$
b_{12}^{c_{11}}=b_{12}^{a_{21}^{-1} a_{12}}
$$

in the form

$$
b_{12}^{c_{11}}=b_{12}^{b_{12} a_{12} a_{21}^{-1}} .
$$

Conjugating both sides of this relation by $b_{21}^{-1}$ and using the fact that $c_{11} b_{21}^{-1}=b_{21}^{-1} c_{11}$ and $b_{12} b_{21}=b_{21} b_{12}$, we get

$$
b_{12}^{c_{11}}=b_{12}^{c_{12} c_{21}^{-1}}
$$

Hence, we have proven

Corollary 5.2. The group $V P_{3}$ is generated by elements

$$
a_{11}, c_{11}, a_{21}, a_{12}, b_{21}, b_{12}
$$

and is defined by relations

$$
\begin{gathered}
{\left[a_{21}, a_{12}\right]=\left[b_{21}, b_{12}\right]=1,} \\
a_{21}^{c_{11}}=a_{21}, b_{21}^{c_{11}}=b_{21}, b_{12}^{c_{11}}=b_{12}^{c_{12} c_{21}^{-1}}, a_{12}^{c_{11}}=a_{12}^{c_{12} c_{21}^{-1}} .
\end{gathered}
$$

Also, we can change the generators $b_{i j}$ to the generators $c_{i j}$.

Corollary 5.3. The group $\mathrm{VP}_{3}$ is generated by elements

$$
a_{11}, c_{11}, a_{21}, a_{12}, c_{21}, c_{12}
$$

and is defined by relations

$$
\begin{gathered}
{\left[a_{21}, a_{12}\right]=\left[c_{21} a_{21}^{-1}, c_{12} a_{12}^{-1}\right]=1} \\
a_{21}^{c_{11}}=a_{21}, c_{21}^{c_{11}}=c_{21}, a_{12}^{c_{11}}=a_{12}^{c_{12} c_{21}^{-1}}, c_{12}^{c_{11}}=c_{12}^{c_{21}^{-1}} .
\end{gathered}
$$

To find a set of defining relations of $T_{2}$ consider a homomorphism $\varphi:\left\langle T_{2}, c_{11}\right\rangle \rightarrow$ $\left\langle c_{11}\right\rangle$ which sends all generators of $T_{2}$ to 1 and sends $c_{11}$ to $c_{11}$. To find the kernel of this homomorphism, we are using the Reidemeister-Schreier method [9, Section 2.3]. The kernel is generated by elements

$$
S_{\lambda, a}=\lambda a \cdot(\overline{\lambda a})^{-1}, \quad \lambda \in\left\langle c_{11}\right\rangle, \quad a \in\left\{a_{12}, a_{21}, b_{12}, b_{21}, c_{11}\right\}
$$

that are equal to

$$
c_{11}^{-k} a_{12} c_{11}^{k}, \quad c_{11}^{-k} a_{21} c_{11}^{k}, \quad c_{11}^{-k} b_{12} c_{11}^{k}, \quad c_{11}^{-k} b_{21} c_{11}^{k}, \quad k \in \mathbb{Z} .
$$

Defining relations of $\operatorname{Ker}(\varphi)$ have the form

$$
c_{11}^{-k} \tau(r) c_{11}^{k}, k \in \mathbb{Z},
$$


where $r$ is a defining relation of the group $\left\langle T_{2}, c_{11}\right\rangle$ and $\tau$ is the rewriteble prosess (see [9, Section 2.3]). If $r$ runs through defining relation which are the conjugation rules, then we can use these defining relations to remove all generators of $\operatorname{Ker}(\varphi)$ and keep only four generators:

$$
a_{12}, a_{21}, b_{12}, b_{21}
$$

It means that the kernel is equal to $T_{2}$. Hence, we have only relations

$$
\left[a_{21}, a_{12}\right]^{c_{11}^{k}}=\left[b_{21}, b_{12}\right]^{c_{11}^{k}}=1, k \in \mathbb{Z} .
$$

We proved

Proposition 5.4. The group $T_{2}$ is generated by elements $a_{12}, a_{21}, b_{12}, b_{21}$ and is defined by relations

$$
\left[a_{21}, a_{12}\right]^{c_{11}^{k}}=\left[b_{21}, b_{12}\right]^{c_{11}^{k}}=1, k \in \mathbb{Z} .
$$

Using the conjugation rules in $V P_{3}$ one can prove

Lemma 5.5. In $V P_{3}$ the following formulas hold

$$
a_{12}^{c_{11}^{k}}=a_{12}^{c_{12}^{k} c_{21}^{-k}}, b_{12}^{c_{11}^{k}}=b_{12}^{c_{12}^{k} c_{21}^{-k}}, k \in \mathbb{Z} .
$$

Using these formulas we can give other description of $T_{2}$.

Proposition 5.6. $T_{2}$ is generated by elements

$$
a_{21}, a_{12}, b_{21}, b_{12}
$$

and is defined by the relations

$$
\left[a_{21}^{c_{21}^{k}}, a_{12}^{c_{12}^{k}}\right]=\left[b_{21}^{c_{21}^{k}}, b_{12}^{c_{12}^{k}}\right]=1, k \in \mathbb{Z} .
$$

Proof. As we know $T_{2}$ is defined by the relations

$$
\left[a_{21}, a_{12}\right]^{c_{11}^{k}}=\left[b_{21}, b_{12}\right]^{c_{11}^{k}}=1 \text {. }
$$

Using Lemma 5.5 and conjugation rules we can rewrite these relations in the form

$$
\left[a_{21}, a_{12}^{c_{12}^{k} c_{21}^{-k}}\right]=\left[b_{21}, b_{12}^{c_{12}^{k} c_{21}^{-k}}\right]=1 .
$$

Conjugating both sides of these relations by $c_{21}^{k}$, we get the need relations.

5.2. $V P_{4}$ and its subgroup $T_{3}$. The group $V P_{4}$ is generated by elements

$$
\lambda_{12}, \lambda_{21}, \lambda_{13}, \lambda_{23}, \lambda_{31}, \lambda_{32}, \lambda_{14}, \lambda_{24}, \lambda_{34}, \lambda_{41}, \lambda_{42}, \lambda_{43} .
$$

On the over side, $V P_{4}=\left\langle T_{1}, T_{2}, T_{3}\right\rangle$, where

$$
T_{1}=\left\langle a_{11}, b_{11}\right\rangle, T_{2}=\left\langle a_{21}, a_{12}, b_{21}, b_{12}\right\rangle, T_{3}=\left\langle a_{31}, a_{22}, a_{13}, b_{31}, b_{22}, b_{13}\right\rangle .
$$

We have found expressions of the new generators $a_{i j}$ and $b_{i j}$ as words in standard generators of $V_{4}$. Find expressions of the old generators as words in the new generators:

$$
\lambda_{12}=a_{11}, \lambda_{21}=b_{11}, \lambda_{13}=a_{12} a_{11}^{-1}, \lambda_{23}=a_{11} a_{12}^{-1} a_{21}, \lambda_{31}=b_{11}^{-1} b_{12}, \lambda_{32}=b_{21} b_{12}^{-1} b_{11},
$$




$$
\begin{gathered}
\lambda_{14}=a_{13} a_{12}^{-1}, \quad \lambda_{24}=a_{12} a_{13}^{-1} a_{22} a_{21}^{-1}, \quad \lambda_{34}=a_{21} a_{22}^{-1} a_{31}, \\
\lambda_{41}=b_{12}^{-1} b_{13}, \quad \lambda_{42}=b_{21}^{-1} b_{22} b_{13}^{-1} b_{12}, \quad \lambda_{43}=b_{31} b_{22}^{-1} b_{21} .
\end{gathered}
$$

To find a presentation of $V P_{4}$ in the new generators, we can act on $V P_{3}$ by degeneracy maps $s_{1}, s_{2}, s_{3}$. We will use a presentation of $V P_{3}$ from Corollary 5.2. Then

1) The group $s_{0}\left(V P_{3}\right)$ is generated by elements

$$
a_{21}, c_{21}, a_{22}, a_{31}, b_{22}, b_{31}
$$

and is defined by relations

$$
\begin{gathered}
{\left[a_{31}, a_{22}\right]=\left[b_{31}, b_{22}\right]=1,} \\
a_{31}^{c_{21}}=a_{31}, b_{31}^{c_{21}}=b_{31}, a_{22}^{c_{21}}=a_{22}^{c_{22} c_{31}^{-1}}, b_{22}^{c_{21}}=b_{22}^{c_{22} c_{31}^{-1}} .
\end{gathered}
$$

2) The group $s_{1}\left(V P_{3}\right)$ is generated by elements

$$
a_{12}, c_{12}, a_{13}, a_{31}, b_{13}, b_{31}
$$

and is defined by relations

$$
\begin{gathered}
{\left[a_{31}, a_{13}\right]=\left[b_{31}, b_{13}\right]=1,} \\
a_{31}^{c_{12}}=a_{31}, b_{31}^{c_{12}}=b_{31}, a_{13}^{c_{12}}=a_{13}^{c_{13} c_{31}^{-1}}, b_{13}^{c_{12}}=b_{13}^{c_{13} c_{31}^{-1}} .
\end{gathered}
$$

3) The group $s_{2}\left(V P_{3}\right)$ is generated by elements

$$
a_{11}, c_{11}, a_{13}, a_{22}, b_{13}, b_{22}
$$

and is defined by relations

$$
\begin{gathered}
{\left[a_{22}, a_{13}\right]=\left[b_{22}, b_{13}\right]=1,} \\
a_{22}^{c_{11}}=a_{22}, b_{22}^{c_{11}}=b_{22}, a_{13}^{c_{11}}=a_{13}^{c_{13} c_{22}^{-1}}, b_{13}^{c_{11}}=b_{13}^{c_{13} c_{22}^{-1}} .
\end{gathered}
$$

The defining relations of the groups $V P_{3}, s_{i}\left(V P_{3}\right), i=0,1,2$, are not the full set of defining relations of $V P_{4}$. We need to add the commutativity relations:

$$
\left[\lambda_{34}^{*}, \lambda_{12}^{*}\right]=\left[\lambda_{24}^{*}, \lambda_{13}^{*}\right]=\left[\lambda_{14}^{*}, \lambda_{23}^{*}\right]=1 \text {, }
$$

where $\lambda_{i j}^{*}$ is any element from the set $\left\{\lambda_{i j}, \lambda_{j i}\right\}$.

To find defining relations of $T_{3}$ we need to understand that relations in $V P_{4}$ give relations in $T_{3}$. To do it we present $V P_{4}$ as HNN-extensions with some base group $G_{4}$ and stable letter $a_{11}$. Hence, the defining relations of $T_{3}$ came from defining relations of $G_{4}$.

We will analize the relations (5.1) and show that six from these relations are conjugation rules by $a_{11}$ and can be used in a presentation of $V P_{4}$ as HNN-extensions and other relations can be write as defining relations in $G_{4}$.

Commutativity relations $\left[\lambda_{34}^{*}, \lambda_{12}^{*}\right]=1$. These relations have the form

$$
\begin{aligned}
& {\left[\lambda_{34}, \lambda_{12}\right]=1 \Leftrightarrow\left[a_{21} a_{22}^{-1} a_{31}, a_{11}\right]=1,} \\
& {\left[\lambda_{34}, \lambda_{21}\right]=1 \Leftrightarrow\left[a_{21} a_{22}^{-1} a_{31}, b_{11}\right]=1,} \\
& {\left[\lambda_{43}, \lambda_{12}\right]=1 \Leftrightarrow\left[b_{31} b_{22}^{-1} b_{21}, a_{11}\right]=1,} \\
& {\left[\lambda_{43}, \lambda_{21}\right]=1 \Leftrightarrow\left[b_{31} b_{22}^{-1} b_{21}, b_{11}\right]=1 .}
\end{aligned}
$$


Write the first and the third relations in the form

$$
\left(a_{21} a_{22}^{-1} a_{31}\right)^{a_{11}}=a_{21} a_{22}^{-1} a_{31},\left(b_{31} b_{22}^{-1} b_{21}\right)^{a_{11}}=b_{31} b_{22}^{-1} b_{21} .
$$

Then from the second and from the fourth relations follows

$$
\left(a_{21} a_{22}^{-1} a_{31}\right)^{c_{11}}=a_{21} a_{22}^{-1} a_{31},\left(b_{31} b_{22}^{-1} b_{21}\right)^{c_{11}}=b_{31} b_{22}^{-1} b_{21} .
$$

In $V P_{3}$ we have relations $a_{21}^{c_{11}}=a_{21}, b_{21}^{c_{11}}=b_{21}$, and in $s_{2}\left(V P_{3}\right)$ we have relations $a_{22}^{c_{11}}=a_{22}, b_{22}^{c_{11}}=b_{22}$. Hence, from (5.2) we get

$$
a_{31}^{c_{11}}=a_{31}, b_{31}^{c_{11}}=b_{31} .
$$

We proved

Lemma 5.7. From the relations $\left[\lambda_{34}^{*}, \lambda_{12}^{*}\right]=1$ in $V P_{4}$ follow formulas of conjugation by $a_{11}$ :

$$
\left(a_{21} a_{22}^{-1} a_{31}\right)^{a_{11}}=a_{21} a_{22}^{-1} a_{31},\left(b_{31} b_{22}^{-1} b_{21}\right)^{a_{11}}=b_{31} b_{22}^{-1} b_{21},
$$

and formulas of conjugation by $c_{11}$ :

$$
a_{31}^{c_{11}}=a_{31}, b_{31}^{c_{11}}=b_{31} .
$$

Commutativity relations $\left[\lambda_{24}^{*}, \lambda_{13}^{*}\right]=1$. These relations have the form

$$
\begin{aligned}
& {\left[\lambda_{24}, \lambda_{13}\right]=1 \Leftrightarrow\left[a_{12} a_{13}^{-1} a_{22} a_{21}^{-1}, a_{12} a_{11}^{-1}\right]=1, }=1 \\
& {\left[\lambda_{24}, \lambda_{31}\right]=1 \Leftrightarrow\left[a_{12} a_{13}^{-1} a_{22} a_{21}^{-1}, b_{11}^{-1} b_{12}\right]=1, } \\
& {\left[\lambda_{42}, \lambda_{13}\right]=1 \Leftrightarrow\left[b_{21}^{-1} b_{22} b_{13}^{-1} b_{12}, a_{12} a_{11}^{-1}\right]=1, } \\
& {\left[\lambda_{42}, \lambda_{31}\right]=1 \Leftrightarrow\left[b_{21}^{-1} b_{22} b_{13}^{-1} b_{12}, b_{11}^{-1} b_{12}\right]=1 . }
\end{aligned}
$$

From the first relation we have the following conjugation formula by $a_{11}$ :

$$
\left(a_{12} a_{13}^{-1} a_{22} a_{21}^{-1}\right)^{a_{11}}=\left(a_{12} a_{13}^{-1} a_{22} a_{21}^{-1}\right)^{a_{12}} .
$$

The second relation has the form

$$
\left(a_{12} a_{13}^{-1} a_{22} a_{21}^{-1}\right)^{b_{11}^{-1}}=\left(a_{12} a_{13}^{-1} a_{22} a_{21}^{-1}\right)^{b_{12}^{-1}} .
$$

Since $b_{i j}^{-1}=a_{i j} c_{i j}^{-1}$ we have

$$
\left(a_{12} a_{13}^{-1} a_{22} a_{21}^{-1}\right)^{a_{11} c_{11}^{-1}}=\left(a_{12} a_{13}^{-1} a_{22} a_{21}^{-1}\right)^{a_{12} c_{12}^{-1}} .
$$

Using (5.3) rewrite this relation in the form

$$
\left(a_{12} a_{13}^{-1} a_{22} a_{21}^{-1}\right)^{a_{12} c_{11}^{-1}}=\left(a_{13}^{-1} a_{22} a_{21}^{-1} a_{12}\right)^{c_{12}^{-1}} .
$$

That is equivalent to the relation

$$
\left(a_{13}^{-1} a_{22}\right)^{c_{12}^{-1}}=\left(a_{13}^{-1} a_{22}\right)^{c_{11}^{-1}}\left(a_{21}^{-1} a_{12}\right)^{c_{11}^{-1}}\left(a_{12}^{-1} a_{21}\right)^{c_{12}^{-1}} .
$$

Similarly, from the third relation

$$
\left(b_{21}^{-1} b_{22} b_{13}^{-1} b_{12}\right)^{a_{11}}=\left(b_{21}^{-1} b_{22} b_{13}^{-1} b_{12}\right)^{a_{12}} .
$$

It is a formula of conjugation by $a_{11}$. 
The fourth relation has the form

$$
\left(b_{21}^{-1} b_{22} b_{13}^{-1} b_{12}\right)^{b_{11}^{-1}}=\left(b_{21}^{-1} b_{22} b_{13}^{-1} b_{12}\right)^{b_{12}^{-1}} .
$$

Using the equality $b_{11}^{-1}=a_{11} c_{11}^{-1}$, rewrite the last relation in the form

$$
\left(b_{21}^{-1} b_{22} b_{13}^{-1} b_{12}\right)^{a_{11} c_{11}^{-1}}=b_{12} b_{21}^{-1} b_{22} b_{13}^{-1},
$$

and using (5.5) we get

$$
\left(b_{21}^{-1} b_{22} b_{13}^{-1} b_{12}\right)^{a_{12} c_{11}^{-1}}=b_{12} b_{21}^{-1} b_{22} b_{13}^{-1} .
$$

Since $a_{12}=b_{12}^{-1} c_{12}$, we have

$$
\left(b_{12} b_{21}^{-1} b_{22} b_{13}^{-1}\right)^{c_{12}}=\left(b_{12} b_{21}^{-1} b_{22} b_{13}^{-1}\right)^{c_{11}} .
$$

This relation is equivalent to

$$
\left(b_{22} b_{13}^{-1}\right)^{c_{12}}=\left(b_{21} b_{12}^{-1}\right)^{c_{12}}\left(b_{12} b_{21}^{-1}\right)^{c_{11}}\left(b_{22} b_{13}^{-1}\right)^{c_{11}} .
$$

Hence, we have

Lemma 5.8. From the relations $\left[\lambda_{24}^{*}, \lambda_{13}^{*}\right]=1$ in $V P_{4}$ follow formulas of conjugation by $a_{11}$ :

$$
\left(a_{12} a_{13}^{-1} a_{22} a_{21}^{-1}\right)^{a_{11}}=\left(a_{12} a_{13}^{-1} a_{22} a_{21}^{-1}\right)^{a_{12}},\left(b_{21}^{-1} b_{22} b_{13}^{-1} b_{12}\right)^{a_{11}}=\left(b_{21}^{-1} b_{22} b_{13}^{-1} b_{12}\right)^{a_{12}},
$$

and formulas of conjugation by $c_{12}^{-1}$ and by $c_{12}$ :

$$
\begin{gathered}
\left(a_{13}^{-1} a_{22}\right)^{c_{12}^{-1}}=\left(a_{13}^{-1} a_{22}\right)^{c_{11}^{-1}}\left(a_{21}^{-1} a_{12}\right)^{c_{11}^{-1}}\left(a_{12}^{-1} a_{21}\right)^{c_{12}^{-1}}, \\
\left(b_{22} b_{13}^{-1}\right)^{c_{12}}=\left(b_{21} b_{12}^{-1}\right)^{c_{12}}\left(b_{12} b_{21}^{-1}\right)^{c_{11}}\left(b_{22} b_{13}^{-1}\right)^{c_{11}} .
\end{gathered}
$$

Let us prove that we can simplify two last relations from this lemma.

Lemma 5.9. In $\mathrm{VP}_{4}$ the following relations hold

$$
\begin{aligned}
& \text { 1) }\left(a_{13}^{-1} a_{22}\right)^{c_{12}^{-1}}=\left(a_{13}^{-1} a_{22}\right)^{c_{11}^{-1}}\left[c_{21}, c_{12}^{-1}\right] \text {, } \\
& \text { 2) }\left(b_{22} b_{13}^{-1}\right)^{c_{12}}=\left[c_{12}, c_{21}^{-1}\right]\left(b_{22} b_{13}^{-1}\right)^{c_{11}} .
\end{aligned}
$$

Proof. 1) To prove the first relation, we need to prove the equality

$$
\left(a_{21}^{-1} a_{12}\right)^{c_{11}^{-1}}\left(a_{12}^{-1} a_{21}\right)^{c_{12}^{-1}}=\left[c_{21}, c_{12}^{-1}\right] .
$$

We have

$$
c_{11}^{-1} a_{12} a_{21}^{-1} c_{12}^{-1}=\left(c_{21}^{-1} c_{21}\right) c_{11}^{-1} a_{12} a_{21}^{-1} c_{12}^{-1},
$$

where we add unit element $1=c_{21}^{-1} c_{21}$.

Since $c_{21} c_{11}^{-1}=c_{11}^{-1} c_{21}$ and $c_{21} a_{21}^{-1} \cdot a_{12} c_{12}^{-1}=a_{12} c_{12}^{-1} \cdot c_{21} a_{21}^{-1}$, the last expression has the form

$$
\begin{gathered}
c_{21}^{-1} c_{11}^{-1}\left(c_{21} a_{21}^{-1} \cdot a_{12} c_{12}^{-1}\right)=c_{21}^{-1} c_{11}^{-1}\left(a_{12} c_{12}^{-1} \cdot c_{21} a_{21}^{-1}\right)=c_{21}^{-1}\left(a_{12} c_{12}^{-1} \cdot c_{21} a_{21}^{-1}\right)^{c_{11}} c_{11}^{-1}= \\
=c_{21}^{-1} a_{12}^{c_{12} c_{21}^{-1}} c_{12}^{-c_{21}^{-1}} c_{21} a_{21}^{-1} c_{11}^{-1}=c_{12}^{-1} a_{12} a_{21}^{-1} c_{11}^{-1} .
\end{gathered}
$$


Hence, we have the relation

$$
c_{11}^{-1} a_{12} a_{21}^{-1} c_{12}^{-1}=c_{12}^{-1} a_{12} a_{21}^{-1} c_{11}^{-1} .
$$

From this relation follows

$$
\begin{gathered}
a_{12} a_{21}^{-1} c_{12}^{-1}=\left(c_{12}^{-1} a_{12} a_{21}^{-1}\right)^{c_{11}^{-1}} \Leftrightarrow\left(a_{12} a_{21}^{-1}\right)^{c_{12}^{-1}}=c_{12} c_{12}^{-c_{11}^{-1}}\left(a_{12} a_{21}^{-1}\right)^{c_{11}^{-1}} \Leftrightarrow \\
\Leftrightarrow\left[c_{21}, c_{12}^{-1}\right]=\left(a_{21}^{-1} a_{12}\right)^{c_{11}^{-1}}\left(a_{12}^{-1} a_{21}\right)^{c_{12}^{-1}} .
\end{gathered}
$$

From the last relation following the first relation in the lemma.

2) Let us prove the equality

$$
\left(b_{21} b_{12}^{-1}\right)^{c_{12}}\left(b_{12} b_{21}^{-1}\right)^{c_{11}}=\left[c_{12}, c_{21}^{-1}\right]
$$

Similarly to the previous case, we have

$$
\begin{gathered}
c_{12} b_{12}^{-1} b_{21} c_{11}=c_{12} b_{12}^{-1} b_{21} c_{11}\left(c_{21}^{-1} c_{21}\right)=\left(c_{12} b_{12}^{-1} \cdot b_{21} c_{21}^{-1}\right) c_{11} c_{21}= \\
\left.\left.=c_{11}\left(b_{21} c_{21}^{-1} \cdot c_{12} b_{12}^{-1}\right)^{c_{11}} c_{21}=c_{11} b_{21} c_{21}^{-1} \cdot c_{12}^{c_{21}^{-1}} b_{12}^{-c_{12} c_{21}^{-1}}\right) c_{21}\right)=c_{11} b_{21} b_{12}^{-1} c_{12} .
\end{gathered}
$$

Hence, we have found the relation

$$
c_{12} b_{12}^{-1} b_{21} c_{11}=c_{11} b_{21} b_{12}^{-1} c_{12} .
$$

From this relation

$$
c_{12}^{c_{11}}\left(b_{12}^{-1} b_{21}\right)^{c_{11}}=b_{21} b_{12}^{-1} c_{12} \Leftrightarrow c_{12}^{-1} c_{12}^{c_{21}^{-1}}\left(b_{12}^{-1} b_{21}\right)^{c_{11}}=\left(b_{21} b_{12}^{-1}\right)^{c_{12}} .
$$

This relation is equivalent to the need relation.

Corollary 5.10. In $\mathrm{VP}_{4}$ the following formulas hold

1) $a_{22}^{c_{12}^{-1}}=a_{13}^{c_{13}^{-1} c_{31}} a_{13}^{-c_{13}^{-1} c_{22}} a_{22}\left[c_{21}, c_{12}^{-1}\right]$

2) $b_{22}^{c_{12}}=\left[c_{12}, c_{21}^{-1}\right] b_{22} b_{13}^{-c_{13} c_{22}^{-1}} b_{13}^{c_{13} c_{31}^{-1}}$

3) $a_{22}^{c_{12}}=\left[c_{12}, c_{21}^{-1}\right] a_{13}^{-c_{13} c_{22}^{-1}} a_{22} a_{13}^{c_{13} c_{31}^{-1}}$,

4) $b_{22}^{c_{12}^{-1}}=b_{13}^{c_{13}^{-1} c_{31}} b_{22} b_{13}^{-c_{13}^{-1} c_{22}}\left[c_{21}, c_{12}^{-1}\right]$.

Proof. 1) Let us prove the first formula. The prove of the second one is the same. Take the first relation in Lemma 5.9.

$$
\left(a_{13}^{-1} a_{22}\right)^{c_{12}^{-1}}=\left(a_{13}^{-1} a_{22}\right)^{c_{11}^{-1}}\left[c_{21}, c_{12}^{-1}\right] .
$$

Using the conjugation formulas, we get

$$
a_{13}^{-c_{13}^{-1} c_{31}} a_{22}^{c_{12}^{-1}}=a_{13}^{-c_{13}^{-1} c_{22}} a_{22}\left[c_{21}, c_{12}^{-1}\right] .
$$

From this relation we get the first formula:

$$
a_{22}^{c_{12}^{-1}}=a_{13}^{c_{13}^{-1} c_{31}} a_{13}^{-c_{13}^{-1} c_{22}} a_{22}\left[c_{21}, c_{12}^{-1}\right] .
$$


3) Let us prove the third formula. The prove of the fourth one is the same. Take the first relation in Lemma 5.9.

$$
\left(a_{13}^{-1} a_{22}\right)^{c_{12}^{-1}}=\left(a_{13}^{-1} a_{22}\right)^{c_{11}^{-1}}\left[c_{21}, c_{12}^{-1}\right] .
$$

Since

$$
\left[c_{21}, c_{12}^{-1}\right]=c_{11} c_{12} c_{11}^{-1} c_{12}^{-1},
$$

then the relation have the form

$$
a_{13}^{-1} a_{22}=c_{12}^{-1} c_{11}\left(a_{13}^{-1} a_{22}\right) c_{12} c_{11}^{-1} .
$$

Conjugating both sides by $c_{11}$ we get

$$
a_{13}^{-c_{11}} a_{22}=\left[c_{11}, c_{12}\right] a_{13}^{-c_{12}} a_{22}^{c_{12}} .
$$

Since $a_{13}$ and $a_{22}$ are commute, then

$$
a_{13}^{-c_{11}} a_{22}=\left[c_{11}, c_{12}\right] a_{22}^{c_{12}} a_{13}^{-c_{12}}
$$

or

Using the formulas

$$
a_{22}^{c_{12}}=\left[c_{12}, c_{11}\right] a_{13}^{-c_{11}} a_{22} a_{13}^{c_{12}} .
$$

$$
\left[c_{12}, c_{11}\right]=\left[c_{12}, c_{21}^{-1}\right], a_{13}^{c_{11}}=a_{13}^{c_{13} c_{22}^{-1}}, a_{13}^{c_{12}}=a_{13}^{c_{13} c_{31}^{-1}},
$$

we get the need relation.

Commutativity relations $\left[\lambda_{14}^{*}, \lambda_{23}^{*}\right]=1$. These relations have the form

$$
\begin{aligned}
& {\left[\lambda_{14}, \lambda_{23}\right]=1 \Leftrightarrow\left[a_{13} a_{12}^{-1}, a_{11} a_{12}^{-1} a_{21}\right]=1,} \\
& {\left[\lambda_{14}, \lambda_{32}\right]=1 \Leftrightarrow\left[a_{13} a_{12}^{-1}, b_{21} b_{12}^{-1} b_{11}\right]=1,} \\
& {\left[\lambda_{41}, \lambda_{23}\right]=1 \Leftrightarrow\left[b_{12}^{-1} b_{13}, a_{11} a_{12}^{-1} a_{21}\right]=1,} \\
& {\left[\lambda_{41}, \lambda_{32}\right]=1 \Leftrightarrow\left[b_{12}^{-1} b_{13}, b_{21} b_{12}^{-1} b_{11}\right]=1 .}
\end{aligned}
$$

The first relation gives the following conjugation rule by $a_{11}$ :

$$
\left(a_{13} a_{12}^{-1}\right)^{a_{11}}=\left(a_{13} a_{12}^{-1}\right)^{a_{21}^{-1} a_{12}} .
$$

The third relation gives the following conjugation rule

$$
\left(b_{12}^{-1} b_{13}\right)^{a_{11}}=\left(b_{12}^{-1} b_{13}\right)^{a_{21}^{-1} a_{12}} .
$$

Since $b_{i j}=c_{i j} a_{i j}^{-1}$, then

$$
b_{21} b_{12}^{-1} b_{11}=c_{21} a_{21}^{-1} a_{12} c_{12}^{-1} c_{11} a_{11}^{-1},
$$

and the second relation:

$$
\left[a_{13} a_{12}^{-1}, b_{21} b_{12}^{-1} b_{11}\right]=\left[a_{13} a_{12}^{-1}, c_{21} a_{21}^{-1} a_{12} c_{12}^{-1} c_{11} a_{11}^{-1}\right]=1
$$

gives the following relation

$$
\left(a_{13} a_{12}^{-1}\right)^{a_{11} c_{11}^{-1} c_{12} a_{12}^{-1} a_{21} c_{21}^{-1}}=a_{13} a_{12}^{-1} .
$$


Since $c_{12} a_{12}^{-1} \cdot a_{21} c_{21}^{-1}=a_{21} c_{21}^{-1} \cdot c_{12} a_{12}^{-1}$, then

$$
\left(a_{13} a_{12}^{-1}\right)^{a_{11} c_{11}^{-1} a_{21} c_{21}^{-1} \cdot c_{12}}=\left(a_{13} a_{12}^{-1}\right)^{a_{12}} .
$$

Using (5.7) rewrite this relation in the form

$$
\left(a_{13} a_{12}^{-1}\right)^{a_{21}^{-1} a_{12} c_{11}^{-1} a_{21} c_{21}^{-1} c_{12}}=\left(a_{13} a_{12}^{-1}\right)^{a_{12}} .
$$

Since $a_{21}^{-1} a_{12}=a_{12} a_{21}^{-1}$, it is equivalent to

$$
\left(a_{12}^{-1} a_{13}\right)^{a_{21}^{-1} c_{11}^{-1} a_{21} c_{21}^{-1} c_{12}}=a_{12}^{-1} a_{13} .
$$

Since $\left[a_{21}, c_{11}\right]=1$, then

$$
\left(a_{12}^{-1} a_{13}\right)^{c_{11}^{-1} c_{21}^{-1} c_{12}}=a_{12}^{-1} a_{13} .
$$

Using a conjugation formula by $c_{11}^{-1}$ we get

$$
\left(a_{12}^{-c_{12}^{-1} c_{21}} a_{13}^{c_{13}^{-1} c_{22}}\right)^{c_{21}^{-1} c_{12}}=a_{12}^{-1} a_{13} .
$$

Hence

$$
a_{13}^{c_{13}^{-1} c_{22}}=a_{13}^{c_{12}^{-1} c_{21}} .
$$

Similarly, the forth relation has the form

$$
\left(b_{12}^{-1} b_{13}\right)^{a_{11} c_{11}^{-1} c_{12} a_{12}^{-1} a_{21} c_{21}^{-1}}=b_{12}^{-1} b_{13} .
$$

Using (5.8) rewrite the forth relations in the form

$$
\left(b_{12}^{-1} b_{13}\right)^{a_{21}^{-1} a_{12} c_{11}^{-1} c_{12} a_{12}^{-1} a_{21} c_{21}^{-1}}=b_{12}^{-1} b_{13} .
$$

Using the relation $c_{12} a_{12}^{-1} \cdot a_{21} c_{21}^{-1}=a_{21} c_{21}^{-1} \cdot c_{12} a_{12}^{-1}$ and $b_{12}^{-1} b_{13}=a_{12} c_{12}^{-1} c_{13} a_{13}^{-1}$ we can present this relation in the form

$$
\left(c_{12}^{-1} c_{13} a_{13}^{-1} a_{12}\right)^{a_{21}^{-1} c_{11}^{-1} a_{21} c_{21}^{-1} c_{12}}=c_{12}^{-1} c_{13} a_{13}^{-1} a_{12} .
$$

Since $\left[a_{21}, c_{11}\right]=1$, we have

$$
\left(c_{12}^{-1} c_{13} a_{13}^{-1} a_{12}\right)^{c_{11}^{-1} c_{21}^{-1} c_{12}}=c_{12}^{-1} c_{13} a_{13}^{-1} a_{12} .
$$

Using the formulas of conjugating by $c_{11}^{-1}$ we get

$$
c_{13}^{c_{22}} a_{13}^{-c_{13}^{-1} c_{22}}=\left(c_{13} a_{13}^{-1}\right)^{c_{12}^{-1} c_{21}} .
$$

Using (5.9) we have

Using a conjugation formula by $c_{12}^{-1}$

$$
c_{13}^{c_{22}}=c_{13}^{c_{12}^{-1} c_{21}} .
$$

$$
c_{13}^{c_{22}}=\left(c_{13}^{c_{31}}\right)^{c_{21}} .
$$

Conjugating both sides by $c_{21}^{-1}$

$$
c_{22}^{-c_{21}^{-1}} c_{13}^{c_{21}^{-1}} c_{22}^{c_{21}^{-1}}=c_{13}^{c_{31}}
$$


Using the conjugation rules by $c_{21}^{-1}$

$$
c_{22}^{-c_{31}} c_{13}^{c_{21}^{-1}} c_{22}^{c_{31}}=c_{13}^{c_{31}}
$$

or

$$
c_{13}^{c_{21}^{-1}}=c_{13}^{c_{22}^{-1} c_{31}} .
$$

Now come back to the relation $(5.9)$ and write it in the form

$$
a_{13}^{c_{13}^{-1} c_{22} c_{21}^{-1}}=a_{13}^{c_{12}^{-1}} .
$$

Using the conjugation formulas, rewrite the left side, we arrive to relation

$$
\left(c_{22}^{-1}\right)^{c_{21}^{-1}} c_{13}^{c_{21}^{-1}} a_{13}^{c_{21}^{-1}} c_{13}^{-c_{21}^{-1}} c_{22}^{c_{21}^{-1}}=a_{13}^{c_{12}^{-1}} .
$$

Using the conjugation rules, we get

$$
c_{22}^{-c_{31}} c_{13}^{c_{22}^{-1} c_{31}} a_{13}^{c_{21}^{-1}} c_{13}^{-c_{22}^{-1} c_{31}} c_{22}^{c_{31}}=a_{13}^{c_{13}^{-1} c_{31}} .
$$

It is equivalent to

$$
a_{13}^{c_{21}^{-1}}=c_{13}^{-c_{22}^{-1} c_{31}} c_{22}^{c_{31}} a_{13}^{c_{13}^{-1} c_{31}} c_{22}^{-c_{31}} c_{13}^{c_{22}^{-1} c_{31}}
$$

and after cancelations

$$
a_{13}^{c_{21}^{-1}}=a_{13}^{c_{22}^{-1} c_{31}}
$$

Since $b_{13}=c_{13} a_{13}^{-1}$, then using the last relation and relation 5.10 , we get

$$
b_{13}^{c_{21}^{-1}}=b_{13}^{c_{22}^{-1} c_{31}} \text {. }
$$

Hence, we proved

Lemma 5.11. The commutativity relations $\left[\lambda_{14}^{*}, \lambda_{23}^{*}\right]=1$ in $V P_{4}$ give the following conjugation formulas by $a_{11}$ :

$$
\left(a_{13} a_{12}^{-1}\right)^{a_{11}}=\left(a_{13} a_{12}^{-1}\right)^{a_{21}^{-1} a_{12}},\left(b_{12}^{-1} b_{13}\right)^{a_{11}}=\left(b_{12}^{-1} b_{13}\right)^{a_{21}^{-1} a_{12}},
$$

and the conjugation formulas by $c_{21}^{-1}$ :

$$
a_{13}^{c_{21}^{-1}}=a_{13}^{c_{22}^{-1} c_{31}}, b_{13}^{c_{21}^{-1}}=b_{13}^{c_{22}^{-1} c_{31}} .
$$

5.3. $V P_{4}$ as $\mathbf{H N N}$-extension. From the relations of commutativity in $V P_{4}$ we got the following conjugation formulas by element $a_{11}$ :

$$
\begin{gathered}
\left(a_{21} a_{22}^{-1} a_{31}\right)^{a_{11}}=a_{21} a_{22}^{-1} a_{31}, \quad\left(b_{31} b_{22}^{-1} b_{21}\right)^{a_{11}}=b_{31} b_{22}^{-1} b_{21}, \\
\left(a_{12} a_{13}^{-1} a_{22} a_{21}^{-1}\right)^{a_{11}}=\left(a_{12} a_{13}^{-1} a_{22} a_{21}^{-1}\right)^{a_{12}},\left(b_{21}^{-1} b_{22} b_{13}^{-1} b_{12}\right)^{a_{11}}=\left(b_{21}^{-1} b_{22} b_{13}^{-1} b_{12}\right)^{a_{12}}, \\
\left(a_{13} a_{12}^{-1}\right)^{a_{11}}=\left(a_{13} a_{12}^{-1}\right)^{a_{21}^{-1} a_{12}},\left(b_{12}^{-1} b_{13}\right)^{a_{11}}=\left(b_{12}^{-1} b_{13}\right)^{a_{21}^{-1} a_{12}} .
\end{gathered}
$$

Denote

$$
A_{a}=\left\langle a_{21} a_{22}^{-1} a_{31}, a_{12} a_{13}^{-1} a_{22} a_{21}^{-1}, a_{13} a_{12}^{-1}\right\rangle .
$$

We see that

$$
A_{a}=\left\langle\lambda_{34}, \lambda_{24}, \lambda_{14}\right\rangle \text {. }
$$


Denote

$$
A_{b}=\left\langle b_{31} b_{22}^{-1} b_{21}, b_{21}^{-1} b_{22} b_{13}^{-1} b_{12}, b_{12}^{-1} b_{13}\right\rangle
$$

We see that

$$
A_{b}=\left\langle\lambda_{43}, \lambda_{42}, \lambda_{41}\right\rangle
$$

Also denote

$$
B_{a}=\left\langle a_{21} a_{22}^{-1} a_{31}, \quad\left(a_{12} a_{13}^{-1} a_{22} a_{21}^{-1}\right)^{a_{12}},\left(a_{13} a_{12}^{-1}\right)^{a_{21}^{-1} a_{12}}\right\rangle=\left\langle\lambda_{34}, \lambda_{24}^{\lambda_{12}}, \quad \lambda_{14}^{\lambda_{12}}\right\rangle
$$

and

$$
B_{b}=\left\langle b_{31} b_{22}^{-1} b_{21},\left(b_{21}^{-1} b_{22} b_{13}^{-1} b_{12}\right)^{a_{12}},\left(b_{12}^{-1} b_{13}\right)^{a_{21}^{-1} a_{12}}\right\rangle=\left\langle\lambda_{43}, \lambda_{42}^{\lambda_{12}}, \lambda_{41}^{\lambda_{12}}\right\rangle .
$$

We see that $B_{a}=A_{a}^{a_{11}}, B_{b}=A_{b}^{a_{11}}$. Put $A=\left\langle A_{a}, A_{b}\right\rangle, B=\left\langle B_{a}, B_{b}\right\rangle$. Since $B$ is conjugate with $A$, then $A$ is isomorphic to $B$ and we get

Theorem 5.12. $\mathrm{VP}_{4}$ is the HNN-extension with the base group

$$
G_{4}=\left\langle c_{11}, a_{21}, a_{12}, c_{21}, c_{12}, a_{31}, a_{22}, a_{13}, b_{31}, b_{22}, b_{13}\right\rangle
$$

associated subgroups $A$ and $B$, stable letter $a_{11} . G_{4}$ is defined by the following relations (here $\varepsilon= \pm 1$ ):

1) conjugations by $c_{11}^{\varepsilon}$

$$
\begin{gathered}
a_{21}^{c_{11}^{\varepsilon}}=a_{21}, a_{12}^{c_{11}^{\varepsilon}}=a_{12}^{c_{12}^{\varepsilon} c_{21}^{-\varepsilon}}, c_{21}^{c_{11}^{\varepsilon}}=c_{21}, c_{12}^{c_{11}^{\varepsilon}}=c_{12}^{c_{21}^{-\varepsilon}}, \\
a_{31}^{c_{11}^{\varepsilon}}=a_{31}, a_{22}^{c_{11}^{\varepsilon}}=a_{22}, a_{13}^{c_{11}^{\varepsilon}}=a_{13}^{c_{13}^{\varepsilon}}{ }_{22}^{-\varepsilon}, b_{31}^{c_{11}^{\varepsilon}}=b_{31}, b_{22}^{c_{11}^{\varepsilon}}=b_{22}, b_{13}^{c_{11}^{\varepsilon}}=b_{13}^{c_{13}^{\varepsilon} c_{22}^{-\varepsilon}},
\end{gathered}
$$

2) conjugations by $c_{21}^{\varepsilon}$

$$
a_{31}^{c_{21}^{\varepsilon}}=a_{31}, a_{22}^{c_{21}^{\varepsilon}}=a_{22}^{c_{22}^{\varepsilon} c_{31}^{-\varepsilon}}, a_{13}^{c_{21}^{\varepsilon}}=a_{13}^{c_{22}^{\varepsilon} c_{31}^{-\varepsilon}}, b_{31}^{c_{21}^{\varepsilon}}=b_{31}, b_{22}^{c_{21}^{\varepsilon}}=b_{22}^{c_{22}^{\varepsilon} c_{31}^{-\varepsilon}}, b_{13}^{c_{21}^{\varepsilon}}=b_{13}^{c_{22}^{\varepsilon}}{ }_{31}^{-\varepsilon}
$$

3) conjugations by $c_{12}^{\varepsilon}$

$$
\begin{gathered}
a_{31}^{c_{12}^{\varepsilon}}=a_{31}, a_{13}^{c_{12}^{\varepsilon}}=a_{13}^{c_{13}^{\varepsilon} c_{31}^{-\varepsilon}}, b_{31}^{c_{12}^{\varepsilon}}=b_{31}, b_{13}^{c_{12}^{\varepsilon}}=b_{13}^{c_{13}^{\varepsilon} c_{31}^{-\varepsilon}} \\
a_{22}^{c_{12}^{-1}}=a_{13}^{c_{13}^{-1} c_{31}} a_{13}^{-c_{13}^{-1} c_{22}} a_{22}\left[c_{21}, c_{12}^{-1}\right], a_{22}^{c_{12}}=\left[c_{12}, c_{21}^{-1}\right] a_{13}^{-c_{13} c_{22}^{-1}} a_{22} a_{13}^{c_{13} c_{31}^{-1}} \\
b_{22}^{c_{12}^{-1}}=b_{13}^{c_{13}^{-1} c_{31}} b_{22} b_{13}^{-c_{13}^{-1} c_{22}}\left[c_{21}, c_{12}^{-1}\right], \quad b_{22}^{c_{12}}=\left[c_{12}, c_{21}^{-1}\right] b_{22} b_{13}^{-c_{13} c_{22}^{-1}} b_{13}^{c_{13} c_{31}^{-1}}
\end{gathered}
$$

4) commutativity relations

$$
\begin{gathered}
{\left[a_{21}, a_{12}\right]=\left[a_{31}, a_{22}\right]=\left[a_{31}, a_{13}\right]=\left[a_{22}, a_{13}\right]=1,} \\
{\left[c_{21} a_{21}^{-1}, c_{12} a_{21}^{-1}\right]=\left[b_{31}, b_{22}\right]=\left[b_{31}, b_{13}\right]=\left[b_{22}, b_{13}\right]=1 .}
\end{gathered}
$$


Hence, to find defining relations of $T_{3}$ we need to study $G_{4}$.

Define the following subgroup of $G_{4}$ :

$$
Q=\left\langle a_{21}, a_{12}, c_{21}, c_{12}, a_{31}, a_{22}, a_{13}, b_{31}, b_{22}, b_{13}\right\rangle .
$$

From relations 1) of Theorem 5.12 follows that $Q$ is normal in $G_{4}$ and is the kernel of the homomorphism

$$
G_{4} \longrightarrow\left\langle c_{11}\right\rangle
$$

which sends $c_{11}$ to $c_{11}$ and sends all other generators to 1 . Similarly to the case $V P_{3}$ one can see that $Q$ is defined by relations which come from relations 2) - 4) of Theorem 5.12 by conjugation $c_{11}^{k}, k \in \mathbb{Z}$. Using the defining relations of $G_{4}$ one can prove that all conjugations of relations 2) -3 ) are equivalent to relations 2) -3 ). Hence,

Lemma 5.13. The group $Q$ is defined by relations 2) - 3) of Theorem 5.12 and relations

$$
\begin{gathered}
{\left[a_{21}, a_{12}\right]^{c_{11}^{k}}=\left[a_{31}, a_{22}\right]^{c_{11}^{k}}=\left[a_{31}, a_{13}\right]^{c_{11}^{k}}=\left[a_{22}, a_{13}\right]^{c_{11}^{k}}=1,} \\
{\left[c_{21} a_{21}^{-1}, c_{12} a_{21}^{-1}\right]^{c_{11}^{k}}=\left[b_{31}, b_{22}\right]^{c_{11}^{k}}=\left[b_{31}, b_{13}\right]^{c_{11}^{k}}=\left[b_{22}, b_{13}\right]^{c_{11}^{k}}=1,}
\end{gathered}
$$

that can be written in the form

$$
\begin{gathered}
{\left[a_{21}, a_{12}^{c_{12}^{k} c_{21}^{-k}}\right]=\left[a_{31}, a_{22}\right]=\left[a_{31}, a_{13}^{c_{13}^{k} c_{22}^{-k}}\right]=\left[a_{22}, a_{13}^{c_{13}^{k} c_{22}^{-k}}\right]=1,} \\
{\left[c_{21} a_{21}^{-1}, c_{12}^{c_{21}^{-k}} a_{21}^{-c_{12}^{k} c_{21}^{-k}}\right]=\left[b_{31}, b_{22}\right]=\left[b_{31}, b_{13}^{c_{13}^{k} c_{22}^{-k}}\right]=\left[b_{22}, b_{13}^{c_{13}^{k} c_{22}^{-k}}\right]=1,}
\end{gathered}
$$

for all integer numbers $k$.

Now we can prove the main result of the present paper.

Theorem 5.14. The group

$$
T_{3}=\left\langle a_{31}, a_{22}, a_{13}, b_{31}, b_{22}, b_{13}\right\rangle
$$

is defined by relations

$$
\begin{gathered}
{\left[a_{31}, a_{22}^{c_{22}^{m} c_{31}^{-m}}\right]=\left[a_{31}, a_{13}^{c_{13}^{k} c_{22}^{m-k} c_{31}^{-m}}\right]=\left[a_{22}^{c_{22}^{m} c_{31}^{-m}}, a_{13}^{c_{13}^{k} c_{22}^{m-k}} c_{31}^{-m}\right]=1,} \\
{\left[b_{31}, b_{22}^{c_{22}^{m} c_{31}^{-m}}\right]=\left[b_{31}, b_{13}^{c_{13}^{k} c_{22}^{m-k}} c_{31}^{-m}\right]=\left[b_{22}^{c_{22}^{m} c_{31}^{-m}}, b_{13}^{c_{13}^{k} c_{22}^{m-k} c_{31}^{-m}}\right]=1 .}
\end{gathered}
$$

where $k, m \in \mathbb{Z}$.

Proof. In Lemma 5.13 we have found a set of defining relations for $Q$. From this set follows that $Q$ is a free product of subgroups $Q_{1}=\left\langle c_{21}, c_{12}, a_{21}, a_{12}\right\rangle$ and $Q_{2}=$ $\left\langle c_{21}, c_{12}, a_{31}, a_{22}, a_{13}, b_{31}, b_{22}, b_{13}\right\rangle$ with amalgamated subgroup $Q_{c}=\left\langle c_{21}, c_{12}\right\rangle$. Hence, we have a set of defining relations for $Q_{2}$ : it includes relations of $Q$, that contains only generators of $Q_{2}$.

Now consider a homomorphism

$$
\varphi: Q_{2} \longrightarrow\left\langle c_{21}\right\rangle
$$

that is defined by the formulas

$$
\varphi\left(c_{21}\right)=c_{21}, \varphi\left(c_{12}\right)=\varphi\left(a_{31}\right)=\varphi\left(a_{22}\right)=\varphi\left(a_{13}\right)=\varphi\left(b_{31}\right)=\varphi\left(b_{22}\right)=\varphi\left(b_{13}\right)=1 .
$$


To find a presentation of $\operatorname{Ker}(\varphi)$ take the set of coset representatives of this kernel in $Q_{2}$ :

$$
\Lambda=\left\{c_{21}^{m} \mid m \in \mathbb{Z}\right\} .
$$

Then $\operatorname{Ker}(\varphi)$ is generated by elements

$$
c_{12}^{c_{21}^{m}}, a_{31}^{c_{21}^{m}}, a_{22}^{c_{21}^{m}}, a_{13}^{c_{21}^{m}}, b_{31}^{c_{21}^{m}}, b_{22}^{c_{21}^{m}}, b_{13}^{c_{21}^{m}} .
$$

Let us denote

$$
d_{m}=c_{12}^{c_{21}^{m}}, m \in \mathbb{Z}
$$

Using the conjugations formulas by $c_{21}$ from Theorem 5.12 , we get

$$
\begin{aligned}
& a_{31}^{c_{21}^{m}}=a_{31}, a_{22}^{c_{21}^{m}}=a_{22}^{c_{22}^{m} c_{31}^{-m}}, a_{13}^{c_{21}^{m}}=a_{13}^{c_{22}^{m} c_{31}^{-m}}, \\
& b_{31}^{c_{21}^{m}}=b_{31}, b_{22}^{c_{21}^{m}}=b_{22}^{c_{22}^{m} c_{31}^{-m}}, b_{13}^{c_{21}^{m}}=b_{13}^{c_{22}^{m} c_{31}^{-m}} .
\end{aligned}
$$

Hence $\operatorname{Ker}(\varphi)$ is generated by elements

$$
d_{m}, m \in \mathbb{Z}, a_{31}, a_{22}, a_{13}, b_{31}, b_{22}, b_{13} .
$$

To find a set of defining relations for $\operatorname{Ker}(\varphi)$ we have to take the following relations in $Q$ :

$$
\begin{gathered}
a_{31}^{c_{12}}=a_{31}, a_{13}^{c_{12}}=a_{13}^{c_{13} c_{31}^{-1}}, b_{31}^{c_{12}}=b_{31}, b_{13}^{c_{12}}=b_{13}^{c_{13} c_{31}^{-1}}, \\
a_{22}^{c_{12}^{-1}}=a_{13}^{c_{13}^{-1} c_{31}} a_{13}^{-c_{13}^{-1} c_{22}} a_{22}\left[c_{21}, c_{12}^{-1}\right], b_{22}^{c_{12}^{-1}}=b_{13}^{c_{13}^{-1} c_{31}} b_{22} b_{13}^{-c_{13}^{-1} c_{22}}\left[c_{21}, c_{12}^{-1}\right], \\
{\left[a_{21}, a_{12}^{c_{12}^{k} c_{21}^{-k}}\right]=\left[a_{31}, a_{22}\right]=\left[a_{31}, a_{13}^{c_{13}^{k} c_{22}^{-k}}\right]=\left[a_{22}, a_{13}^{c_{13}^{k} c_{22}^{-k}}\right]=1,} \\
{\left[c_{21} a_{21}^{-1}, c_{12}^{c_{21}^{-k}} a_{21}^{-c_{12}^{k} c_{21}^{-k}}\right]=\left[b_{31}, b_{22}\right]=\left[b_{31}, b_{13}^{c_{13}^{k}} c_{22}^{-k}\right]=\left[b_{22}, b_{13}^{c_{13}^{k} c_{22}^{-k}}\right]=1,}
\end{gathered}
$$

and conjugate them by $c_{21}^{m}$.

At first consider the relations

$$
a_{22}^{c_{12}^{-1}}=a_{13}^{c_{13}^{-1} c_{31}} a_{13}^{-c_{13}^{-1} c_{22}} a_{22}\left[c_{21}, c_{12}^{-1}\right], b_{22}^{c_{12}^{-1}}=b_{13}^{c_{13}^{-1} c_{31}} b_{22} b_{13}^{-c_{13}^{-1} c_{22}}\left[c_{21}, c_{12}^{-1}\right] .
$$

These relations are equivalent to the following relations from Lemma 5.9

$$
\begin{aligned}
\left(a_{13}^{-1} a_{22}\right)^{c_{12}^{-1}} & =\left(a_{13}^{-1} a_{22}\right)^{c_{11}^{-1}}\left[c_{21}, c_{12}^{-1}\right], \\
\left(b_{22} b_{13}^{-1}\right)^{c_{12}} & =\left[c_{12}, c_{21}^{-1}\right]\left(b_{22} b_{13}^{-1}\right)^{c_{11}} .
\end{aligned}
$$

Using the formulas of conjugations by $c_{11}^{-1}$, rewrite the relation 5.11 in the form

$$
d_{0} a_{13}^{-1} a_{22}=a_{13}^{-c_{13}^{-1} c_{22}} a_{22} d_{1} .
$$

Conjugated it by $c_{21}^{m}$ we get

$$
d_{m} a_{13}^{-c_{22}^{m} c_{31}^{-m}} a_{22}^{c_{22}^{m} c_{31}^{-m}}=a_{13}^{-c_{13}^{-1} c_{22}^{m} c_{22} c_{31}^{-m}} a_{22}^{c_{22}^{m} c_{31}^{-m}} d_{m+1} .
$$

Conjugated both sides of this relation by $c_{31}^{m}$ we have

$$
d_{m}\left(a_{13}^{-1} a_{22}\right)^{c_{22}^{m}}=a_{13}^{-c_{13}^{-1} c_{22}^{m+1}} a_{22}^{c_{22}^{m}} d_{m+1}
$$


From these relations we have

$$
\begin{aligned}
& d_{m+1}=\left(a_{22}^{-1} a_{13}^{c_{13}^{-1} c_{22}}\right)^{c_{22}^{m}} d_{m}\left(a_{13}^{-1} a_{22}\right)^{c_{22}^{m}} \text { for } m \geq 0, \\
& d_{m}=\left(a_{13}^{-c_{13}^{-1} c_{22}} a_{22}\right)^{c_{22}^{m}} d_{m+1}\left(a_{22}^{-1} a_{13}\right)^{c_{22}^{m}} \text { for } m<0 .
\end{aligned}
$$

Analogously, from 5.12 we get the following formulas

$$
\begin{aligned}
& d_{m}=\left(b_{13} b_{22}^{-1}\right)^{c_{22}^{m}} d_{m-1}\left(b_{22} b_{13}^{-c_{13} c_{22}^{-1}}\right)^{c_{22}^{m}} \text { for } m \geq 1 \\
& d_{m-1}=\left(b_{22} b_{13}^{-1}\right)^{c_{22}^{m}} d_{m}\left(b_{13}^{c_{13} c_{22}^{-1}} b_{22}^{-1}\right)^{c_{22}^{m}} \text { for } m<1 .
\end{aligned}
$$

For further calculations introduce the notations

$$
\begin{aligned}
& A_{1}^{(l)}=\left(a_{22}^{-1} a_{13}^{c_{13}^{-1} c_{22}}\right)^{c_{22}^{l}}, A_{2}^{(l)}=\left(a_{13}^{-1} a_{22}\right)^{c_{22}^{l}}, \bar{A}_{i}^{(l)}=\left(A_{i}^{(l)}\right)^{-1} \\
& B_{1}^{(l)}=\left(b_{22} b_{13}^{-c_{13} c_{22}^{-1}}\right)^{c_{22}^{l}}, B_{2}^{(l)}=\left(b_{13} b_{22}^{-1}\right)^{c_{22}^{l}}, \bar{B}_{i}^{(l)}=\left(B_{i}^{(l)}\right)^{-1}
\end{aligned}
$$

for all integers $l$.

Using these notations we express $d_{m}, m \neq 0$ as words, which depend only on $d_{0}^{ \pm 1}$ and other generators of $\operatorname{Ker}(\varphi)$. Using induction on $m$ we get:

for $m \geq 1$

$$
\begin{aligned}
d_{m} & =A_{1}^{(m)} A_{1}^{(m-1)} \ldots A_{1}^{(0)} d_{0} A_{2}^{(0)} A_{2}^{(1)} \ldots A_{2}^{(m)} \\
d_{m} & =B_{2}^{(m)} B_{2}^{(m-1)} \ldots B_{2}^{(1)} d_{0} B_{1}^{(1)} B_{1}^{(2)} \ldots B_{1}^{(m)}
\end{aligned}
$$

for $m \leq-1$

$$
\begin{gathered}
d_{m}=\bar{A}_{1}^{(m)} \bar{A}_{1}^{(m+1)} \ldots \bar{A}_{1}^{(-1)} d_{0} \bar{A}_{2}^{(-1)} \bar{A}_{2}^{(-2)} \ldots \bar{A}_{2}^{(m)} \\
d_{m}=\bar{B}_{2}^{(m+1)} \bar{B}_{2}^{(m+2)} \ldots \bar{B}_{2}^{(0)} d_{0} \bar{B}_{1}^{(0)} \bar{B}_{1}^{(-1)} \ldots \bar{B}_{1}^{(m+1)} .
\end{gathered}
$$

We see that left sides of these relations are equal, then equality of the right sides gives relations:

for $m \geq 1$

$$
\begin{aligned}
d_{0}^{-1} & \left(\bar{A}_{1}^{(0)} \bar{A}_{1}^{(1)} \ldots \bar{A}_{1}^{(m)} \cdot B_{2}^{(m)} B_{2}^{(m-1)} \ldots B_{2}^{(1)}\right) d_{0}= \\
& =A_{2}^{(0)} A_{2}^{(1)} \ldots A_{2}^{(m)} \cdot \bar{B}_{1}^{(m)} \bar{B}_{1}^{(m-1)} \ldots \bar{B}_{1}^{(1)}
\end{aligned}
$$

for $m \leq-1$

$$
\begin{gathered}
d_{0}^{-1}\left(A_{1}^{(-1)} A_{1}^{(-2)} \ldots A_{1}^{(m)} \cdot \bar{B}_{2}^{(m+1)} \bar{B}_{2}^{(m+2)} \ldots \bar{B}_{2}^{(0)}\right) d_{0}= \\
=\bar{A}_{2}^{(-1)} \bar{A}_{2}^{(-2)} \ldots \bar{A}_{2}^{(m)} \cdot \bar{B}_{1}^{(m+1)} \bar{B}_{1}^{(m+2)} \ldots \bar{B}_{1}^{(0)} .
\end{gathered}
$$

Let us consider other relations. 
1) Take the relation $c_{12}^{-1} a_{31} c_{12}=a_{31}$. Conjugating it by $c_{21}^{m}$ we get $d_{m}^{-1} a_{31} d_{m}=a_{31}$. Put instead $d_{m}$ its expressions we get:

and

$$
\begin{aligned}
& d_{0}^{-1}\left(\bar{A}_{1}^{(0)} \bar{A}_{1}^{(1)} \ldots \bar{A}_{1}^{(m)} a_{31} A_{1}^{(m)} A_{1}^{(m-1)} \ldots A_{1}^{(0)}\right) d_{0}= \\
= & A_{2}^{(0)} A_{2}^{(1)} \ldots A_{2}^{(m)} a_{31} \bar{A}_{2}^{(m)} \bar{A}_{2}^{(m-1)} \ldots \bar{A}_{2}^{(0)}, \text { for } m \geq 1,
\end{aligned}
$$

$$
\begin{aligned}
& d_{0}^{-1}\left(A_{1}^{(-1)} A_{1}^{(-2)} \ldots A_{1}^{(m)} a_{31} \bar{A}_{1}^{(m)} \bar{A}_{1}^{(m+1)} \ldots \bar{A}_{1}^{(-1)}\right) d_{0}= \\
= & \bar{A}_{2}^{(-1)} \bar{A}_{2}^{(-2)} \ldots \bar{A}_{2}^{(m)} a_{31} A_{2}^{(m)} A_{2}^{(m+1)} \ldots A_{2}^{(-1)}, \text { for } m \leq-1 .
\end{aligned}
$$

Analogously, from relation $c_{12}^{-1} b_{31} c_{12}=b_{31}$ we get relations

$$
\begin{aligned}
& d_{0}^{-1}\left(\bar{B}_{2}^{(1)} \bar{B}_{2}^{(2)} \ldots \bar{B}_{2}^{(m)} b_{31} B_{2}^{(m)} B_{2}^{(m-1)} \ldots B_{2}^{(1)}\right) d_{0}= \\
= & B_{1}^{(1)} B_{1}^{(2)} \ldots B_{1}^{(m)} b_{31} \bar{B}_{1}^{(m)} \bar{B}_{1}^{(m-1)} \ldots \bar{B}_{1}^{(1)}, \text { for } m \geq 1,
\end{aligned}
$$

and

$$
\begin{aligned}
& d_{0}^{-1}\left(B_{2}^{(0)} B_{2}^{(-1)} \ldots B_{2}^{(m+1)} b_{31} \bar{B}_{2}^{(m+1)} \bar{B}_{2}^{(m+2)} \ldots \bar{B}_{2}^{(0)}\right) d_{0}= \\
= & \bar{B}_{1}^{(0)} \bar{B}_{1}^{(-1)} \ldots \bar{B}_{1}^{(m+1)} b_{31} B_{1}^{(m+1)} B_{1}^{(m+2)} \ldots B_{1}^{(0)}, \text { for } m \leq-1 .
\end{aligned}
$$

2) Take the relation

$$
a_{13}^{c_{12}}=a_{13}^{c_{13} c_{31}^{-1}}
$$

Conjugating it by $c_{21}^{m}$ we get

$$
d_{m}^{-1} a_{13}^{c_{22}^{m}} d_{m}=a_{13}^{c_{13} c_{22}^{m} c_{31}^{-1}} .
$$

Put instead $d_{m}$ its expressions we get:

$$
\begin{aligned}
& d_{0}^{-1}\left(\bar{A}_{1}^{(0)} \bar{A}_{1}^{(1)} \ldots \bar{A}_{1}^{(m)} a_{13}^{c_{22}^{m}} A_{1}^{(m)} A_{1}^{(m-1)} \ldots A_{1}^{(0)}\right) d_{0}= \\
= & A_{2}^{(0)} A_{2}^{(1)} \ldots A_{2}^{(m)} a_{13}^{c_{13} c_{22}^{m} c_{31}^{-1}} \bar{A}_{2}^{(m)} \bar{A}_{2}^{(m-1)} \ldots \bar{A}_{2}^{(0)}, \text { for } m \geq 1,
\end{aligned}
$$

and

$$
\begin{gathered}
d_{0}^{-1}\left(A_{1}^{(-1)} A_{1}^{(-2)} \ldots A_{1}^{(m)} a_{13}^{c_{22}^{m}} \bar{A}_{1}^{(m)} \bar{A}_{1}^{(m+1)} \ldots \bar{A}_{1}^{(-1)}\right) d_{0}= \\
=\bar{A}_{2}^{(-1)} \bar{A}_{2}^{(-2)} \ldots \bar{A}_{2}^{(m)} a_{13}^{c_{13} c_{22}^{m} c_{31}^{-1}} A_{2}^{(m)} A_{2}^{(m+1)} \ldots A_{2}^{(-1)}, \text { for } m \leq-1 .
\end{gathered}
$$

Analogously, the relation

is equivalent to the relations

$$
b_{31}^{c_{12}}=b_{31}^{c_{13} c_{31}^{-1}}
$$

$$
\begin{aligned}
& d_{0}^{-1}\left(\bar{B}_{2}^{(1)} \bar{B}_{2}^{(2)} \ldots \bar{B}_{2}^{(m)} b_{13}^{c_{22}^{m}} B_{2}^{(m)} B_{2}^{(m-1)} \ldots B_{2}^{(1)}\right) d_{0}= \\
& =B_{1}^{(1)} B_{1}^{(2)} \ldots B_{1}^{(m)} b_{13}^{c_{13} c_{22}^{m} c_{31}^{-1}} \bar{B}_{1}^{(m)} \bar{B}_{1}^{(m-1)} \ldots \bar{B}_{1}^{(1)} \text {, for } m \geq 1 \text {, }
\end{aligned}
$$

and

$$
d_{0}^{-1}\left(B_{2}^{(0)} B_{2}^{(-1)} \ldots B_{2}^{(m+1)} b_{13}^{c_{22}^{m}} \bar{B}_{2}^{(m+1)} \bar{B}_{2}^{(m+2)} \ldots \bar{B}_{2}^{(0)}\right) d_{0}=
$$




$$
=\bar{B}_{1}^{(0)} \bar{B}_{1}^{(-1)} \ldots \bar{B}_{1}^{(m+1)} b_{13}^{c_{13} c_{22}^{m} c_{31}^{-1}} B_{1}^{(m+1)} B_{1}^{(m+2)} \ldots B_{1}^{(0)} \text {, for } m \leq-1 .
$$

3) Conjugating the commutativity relations by $c_{21}^{m}$ we get

$$
\begin{gathered}
{\left[a_{31}, a_{22}^{c_{22}^{m} c_{31}^{-m}}\right]=\left[a_{31}, a_{13}^{c_{13}^{k} c_{22}^{m-k} c_{31}^{-m}}\right]=\left[a_{22}^{c_{22}^{m} c_{31}^{-m}}, a_{13}^{c_{13}^{k} c_{22}^{m-k} c_{31}^{-m}}\right]=1,} \\
{\left[b_{31}, b_{22}^{c_{22}^{m} c_{31}^{-m}}\right]=\left[b_{31}, b_{13}^{c_{13}^{k} c_{22}^{m-k}} c_{31}^{-m}\right]=\left[b_{22}^{c_{22}^{m} c_{31}^{-m}}, b_{13}^{c_{13}^{k} c_{22}^{m-k} c_{31}^{-m}}\right]=1 .}
\end{gathered}
$$

Now we will show that $\operatorname{Ker}(\varphi)$ is an HNN-extension with base group $T_{3}$ and stable letter $d_{0}$. Introduce subgroups $A$ and $B$ in $\operatorname{Ker}(\varphi)$. Subgroup $A$ is generated by elements:

for $m \geq 1$

$$
\begin{gathered}
\bar{A}_{1}^{(0)} \bar{A}_{1}^{(1)} \ldots \bar{A}_{1}^{(m)} \cdot B_{2}^{(m)} B_{2}^{(m-1)} \ldots B_{2}^{(1)}, \\
\bar{A}_{1}^{(0)} \bar{A}_{1}^{(1)} \ldots \bar{A}_{1}^{(m)} X_{1} A_{1}^{(m)} A_{1}^{(m-1)} \ldots A_{1}^{(0)}, \text { where } X_{1} \in\left\{a_{31}, a_{31}^{c_{22}^{m}}\right\}, \\
\bar{B}_{2}^{(1)} \bar{B}_{2}^{(2)} \ldots \bar{B}_{2}^{(m)} Y_{1} B_{2}^{(m)} B_{2}^{(m-1)} \ldots B_{2}^{(1)}, \text { where } Y_{1} \in\left\{b_{31}, b_{31}^{c_{22}^{m}}\right\},
\end{gathered}
$$

for $m \leq-1$

$$
\begin{gathered}
A_{1}^{(-1)} A_{1}^{(-2)} \ldots A_{1}^{(m)} \cdot \bar{B}_{2}^{(m+1)} \bar{B}_{2}^{(m+2)} \ldots \bar{B}_{2}^{(0)}, \\
A_{1}^{(-1)} A_{1}^{(-2)} \ldots A_{1}^{(m)} X_{1} \bar{A}_{1}^{(m)} \bar{A}_{1}^{(m+1)} \ldots \bar{A}_{1}^{(-1)}, \\
B_{2}^{(0)} B_{2}^{(-1)} \ldots B_{2}^{(m+1)} Y_{1} \bar{B}_{2}^{(m+1)} \bar{B}_{2}^{(m+2)} \ldots \bar{B}_{2}^{(0)} .
\end{gathered}
$$

Subgroup $B$ is generated by elements:

for $m \geq 1$

$$
\begin{aligned}
& \bar{A}_{2}^{(-1)} \bar{A}_{2}^{(-2)} \ldots \bar{A}_{2}^{(m)} \cdot \bar{B}_{1}^{(m+1)} \bar{B}_{1}^{(m+2)} \ldots \bar{B}_{1}^{(0)}, \\
& A_{2}^{(0)} A_{2}^{(1)} \ldots A_{2}^{(m)} X_{2} \bar{A}_{2}^{(m)} \bar{A}_{2}^{(m-1)} \ldots \bar{A}_{2}^{(0)} \text {, where } X_{2} \in\left\{a_{31}, a_{13}^{c_{13} c_{22}^{m} c_{31}^{-1}}\right\} \text {, } \\
& B_{1}^{(1)} B_{1}^{(2)} \ldots B_{1}^{(m)} b_{13}^{c_{13} c_{22}^{m} c_{31}^{-1}} \bar{B}_{1}^{(m)} \bar{B}_{1}^{(m-1)} \ldots \bar{B}_{1}^{(1)} \text {, where } Y_{2} \in\left\{b_{31}, b_{13}^{c_{13} c_{22}^{m} c_{31}^{-1}}\right\} \text {, }
\end{aligned}
$$

for $m \leq-1$

$$
\begin{gathered}
\bar{A}_{2}^{(-1)} \bar{A}_{2}^{(-2)} \ldots \bar{A}_{2}^{(m)} \cdot \bar{B}_{1}^{(m+1)} \bar{B}_{1}^{(m+2)} \ldots \bar{B}_{1}^{(0)} \\
\bar{A}_{2}^{(-1)} \bar{A}_{2}^{(-2)} \ldots \bar{A}_{2}^{(m)} X_{2} A_{2}^{(m)} A_{2}^{(m+1)} \ldots A_{2}^{(-1)} \\
\bar{B}_{1}^{(0)} \bar{B}_{1}^{(-1)} \ldots \bar{B}_{1}^{(m+1)} Y_{2} B_{1}^{(m+1)} B_{1}^{(m+2)} \ldots B_{1}^{(0)} .
\end{gathered}
$$

The isomorphism $\psi: A \rightarrow B$ is defined conjugation by $d_{0}$ and we see that all relations of $\operatorname{Ker}(\varphi)$, exclude the commutativity relations from 3), define this conjugation. Hence, $\operatorname{Ker}(\varphi)$ is an HNN-extension with base group $T_{3}$, stable letter $d_{0}$ and assotiated subgroups $A$ and $B$ :

$$
\operatorname{Ker}(\varphi)=\left\langle T_{3}, d_{0} \mid \operatorname{rel}\left(T_{3}\right), d_{0}^{-1} A d_{0}=B, \psi\right\rangle .
$$

From the properties of HNN-extension follows that the set of defining relations $\operatorname{rel}\left(T_{3}\right)$ is the set of commutativity relations from 3 ). 


\section{REFERENCES}

[1] V. G. Bardakov, The virtual and universal braids, Fund. Math., 181(2004), 1-18.

[2] V. G. Bardakov, R. Mikhailov, V. V. Vershinin and J. Wu, On the pure virtual braid group $P V_{3}$, Commun. in Algebra, 44, no. 3 (2016), 1350-1378.

[3] V. G. Bardakov, R. Mikhailov and J. Wu, Lifting theorem for the virtual braid groups, preprint in preparation.

[4] D. Bar-Natan, On associators and the Grothendieck-Teichmuller group. I. (English summary), Selecta Math. (N.S.) 4 (1998), no. 2, 183-212.

[5] A. J. Berrick, F. R. Cohen, Y. L. Wong, and J. Wu, Configurations, braids and homotopy groups, J. Amer. Math. Soc, 19, no. 2 (2006), 265-326.

[6] C. Blanchet, I Marin, Cabling Burau representation, ArXiv, 2007.

[7] F. R. Cohen, J. Wu, Artin's braid groups, free groups, and the loop space of the 2-sphere, Q. J. Math., 62, no. 4 (2011), 891-921.

[8] Fengchun Lei, Fengling Li and Jie Wu, On simplicial resolutions of framed links, Trans. Amer. Math. Soc. 366 (2014), no. 6, 3075-3093.

[9] W. Magnus, A. Karrass, D. Solitar, Combinatorial group theory. Presentations of groups in terms of generators and relations. 2nd rev. ed. Dover Books on Advanced Mathematics. New York: Dover Publications, Inc. XII, 444 p. (1976).

[10] R. Mikhailov and I. B. S. Passi, Lower Central and Dimension Series of Groups, Lecture Notes in Mathematics, 1952, Springer-Verlag Berlin Heidelberg, 2009.

Sobolev Institute of Mathematics, Novosibirsk 630090, Russia,

Novosibirsk State University, Novosibirsk 630090, Russia,

Novosibirsk State Agrarian University, Dobrolyubova street, 160, Novosibirsk 630039, RUSSIA,

E-mail address: bardakov@math.nsc.ru

Department of Mathematics, National University of Singapore, 10 Lower Kent Ridge RoAd, Singapore 119076

E-mail address: matwuj@nus.edu.sg

$U R L$ : www.math.nus.edu.sg/ matwujie 